Supplemental information for:

\title{
Copper-Catalyzed Synthesis of Enantioenriched Tetraarylethanes
}

\author{
Wendy S. Jen*, Matthew Truppo, Deborah Amos, Paul Devine, \\ Michael McNevin, Mirlinda Biba, and Kevin R. Campos \\ Department of Process Research, Merck Research Laboratories, P.O. Box 2000, \\ Rahway, New Jersey 07065
}

General Information. Commercial grade reagents were purchased and used without further purification. Organic solutions were concentrated under reduced pressure on a Buchi rotary evaporator. Chromatographic purification of products was accomplished using forcedflow chromatography on Sigma grade 9385 silica mesh 230-400 according to the method described by Still. ${ }^{1}$ Thin-layer chromatography (TLC) was performed on EMD $0.25 \mathrm{~mm}$ silica gel 60-F plates. Visualization of the developed chromatogram was performed by florescence quenching.

The ketoreductase (KRED) enzymes, glucose dehydrogenase (GDH-103) and NADP were purchased from Biocatalytics Inc. (Pasadena, CA). Enzymatic reaction conversion was monitored by analytical high performance liquid chromatography (HPLC) at $215 \mathrm{~nm}$ using an Agilent 1100 series HPLC and a Zorbax Eclipse XDB-C18 (50 x $4.6 \mathrm{~mm})$ column with a flow rate of $1 \mathrm{~mL} / \mathrm{min}(50 \%$ acetonitrile / 50\% water) for 10 minutes. Enantiomeric excess for the enzymatic reactions were was determined using a Berger SFC and a Chiralpak AD-H (250 x $4.6 \mathrm{~mm})$ column with a flow rate of $1.5 \mathrm{~mL} / \mathrm{min}(25 \% \mathrm{MeOH}$ modifier, $215 \mathrm{~nm}, 200 \mathrm{bar}, 35$ $\left.{ }^{\circ} \mathrm{C}\right)$ unless otherwise stated.

${ }^{1} \mathrm{H}$ and ${ }^{13} \mathrm{C}$ spectra were recorded on Bruker AM-400 (400 MHz and $100 \mathrm{MHz}$, respectively), Bruker DRX-500 (500 MHz and $125 \mathrm{MHz}$, respectively) instruments, as noted, and are internally referenced to residual protio solvent signals. Data for ${ }^{1} \mathrm{H}$ are reported with chemical shift $(\delta \mathrm{ppm})$, multiplicity $(\mathrm{s}=$ singlet, $\mathrm{d}=$ doublet, $\mathrm{t}=$ triplet, $\mathrm{q}=$ quartet, $\mathrm{m}=$ multiplet), integration, coupling constant $(\mathrm{Hz})$, and assignment. Data for ${ }^{13} \mathrm{C}$ are reported with chemical shift. Optical Rotations were recorded on a Jasco P-1010 polarimeter (WI

\footnotetext{
${ }^{1}$ Still, W.C.; Kahn, M.; Mitra, A. J. J. Org. Chem. 1978, 43, 2923.
} 
lamp, $589 \mathrm{~nm}, 25^{\circ} \mathrm{C}$ ). Mass spectra (high resolution) were obtained from Agilent LC/MSD TOF. Chiral HPLC analysis was performed on a Berger SFC at 215nm using Chiralcel columns. Dimerization reactions were monitored and diastereomeric ratios were determined using an Agilent 1100 series HPLC and a ACE-C18 column under the following conditions: flow rate of $0.75 \mathrm{ml} / \mathrm{min}, 35 \mathrm{deg}$ Celsius, $60 \mathrm{~min}$ total time, 95:5 to 0:100 AQ/ORG over 50 min. Stock solution: 2L water, $25.2 \mathrm{~g}$ ammonium formate, $15.8 \mathrm{ml}$ formic acid. AQ: 990ml water, $10 \mathrm{ml}$ stock solution. ORG: $900 \mathrm{ml}$ acetonitile, $90 \mathrm{ml}$ water, $10 \mathrm{ml}$ stock solution.

General Procedure A: Ketone Reduction. The ketone reductions were run in 100 $\mathrm{mM}$ potassium phosphate buffer using the following conditions and concentrations: $30^{\circ} \mathrm{C}, \mathrm{pH}$ 7.0, 2 g/L ketoreductase (KRED) enzyme, 2 g/L glucose dehydrogenase (GDH-103), 10 g/L glucose, $1 \mathrm{~g} / \mathrm{L}$ NADP cofactor, $5 \mathrm{~g} / \mathrm{L}$ ketone, 10\% v/v tetrahydrofuran (THF). The enzymes, cofactor and glucose were first dissolved in the buffer. Next, the substrate was dissolved in the THF and the substrate solution was then added to the reaction mixture. The reactions were run for 24 hours at $30{ }^{\circ} \mathrm{C}$ with automatic $\mathrm{pH}$ control using $2 \mathrm{~N} \mathrm{NaOH}$. Upon completion, the reaction solution was extracted with $2 \mathrm{X}$ volumes of ethyl acetate. The organic phase was washed with $10 \mathrm{~mL}$ DI water and then dried using vacuum distillation. The resulting oil or solid was then puried by column chromatography or recrystallized using the specified solvents.

General Procedure B: Dimerization Reaction. To a cooled solution $\left(0^{\circ} \mathrm{C}\right)$ of alcohol (1.0 equiv) in THF $(0.5 \mathrm{M})$ was added $\mathrm{ClP}(\mathrm{O})(\mathrm{OEt}) 2$ (1.2 equiv) dropwise, followed by $\mathrm{PrMgCl}(2.0 \mathrm{M}$ in THF, 1.3 equiv). The reaction was warmed to room temperature an aged until phosphonate ester formation was complete by HPLC. The reaction mixture was then cooled back to $0^{\circ} \mathrm{C}$. $\mathrm{CuCN}$ (0.05 equivalents, unless stated otherwise), MTBE (0.75M relative to alcohol), and $\mathrm{PrMgCl}$ (2.0 equiv) were then added sequentially. The reaction was aged for $15 \mathrm{~min}$ at $0^{\circ} \mathrm{C}$, then warmed to room temperature. Upon completion of the reaction (after aging approximately 30-60 minutes, unless otherwise stated), the reaction was quenched with saturated aqueous ammonium chloride solution, and extracted $3 \mathrm{X}$ with EtOAc. The combined organics were washed with brine, dried over sodium sulfate, and concentrated. The crude oil/solids were then purified by flash chromatography with 5 to $15 \%$ EtOAc/Hexanes. 
(R)-(6-bromopyridin-2-yl)(phenyl)methanol (12). Prepared according to general procedure A from the corresponding ketone $(6.0 \mathrm{~g}, 22.8 \mathrm{mmol})$ using KRED19 to provide alcohol $12(80 \%$ ee). The crude product was then recrystallized to upgrade the ee from $\mathrm{MTBE} / \mathrm{h}$ exanes to provide the title compound as a white solid in 53\% yield (3.22g), $>99 \%$ ee. ${ }^{1} \mathrm{H}$ NMR (400 MHz, $\left.\mathrm{CDCl}_{3}\right) \delta 7.50(\mathrm{t}, J=7.7 \mathrm{~Hz}, 1 \mathrm{H}, \mathrm{pyH}), 7.41-7.27\left(\mathrm{~m}, 6 \mathrm{H}, \mathrm{C}_{6} \mathbf{H}_{5}, \mathrm{pyH}\right)$, 7.14 (d, $J=7.5 \mathrm{~Hz}, 1 \mathrm{H}$, pyH), 5.76 (s, 1H, PhCHOHpy); ${ }^{13} \mathrm{C}$ NMR $\left(100 \mathrm{MHz}, \mathrm{CDCl}_{3}\right) \delta$ 163.0, 142.3, 140.9, 139.2, 128.7, 128.2, 127.1, 126.9, 120.1, 75.1; HRMS (MALDI-TOF) exact mass calculated for $\left(\mathrm{C}_{12} \mathrm{H}_{11} \mathrm{BrNO}\right)$ requires $\mathrm{m} / \mathrm{z} 264.00185$, found $\mathrm{m} / \mathrm{z} 264.00223$ $(\mathrm{M}+\mathrm{H})^{+} ;[\alpha]_{\mathrm{D}}=-9.86^{\circ}\left(\mathrm{c}=1.0, \mathrm{CHCl}_{3}\right)$. Melting point $108.9^{\circ} \mathrm{C}$ to $109.7^{\circ} \mathrm{C}$. Enantiomeric ratios were determined by SFC with a Chiralcel OF column $(250 \times 4.6 \mathrm{~mm} 10 \mu \mathrm{m}$ SFC, isocratic $\left.15 \% \mathrm{MeOH} / \mathrm{CO}_{2}, 1.5 \mathrm{~mL} / \mathrm{min}, 35^{\circ} \mathrm{C}, 200 \mathrm{bar}, 215 \mathrm{~nm}, 15 \mathrm{~min}\right) ; \mathrm{t}_{\mathrm{r}}=7.09 \mathrm{~min}$ and $8.29 \mathrm{~min}$.

\section{Single-Crystal X-Ray Crystallography of 12}

The structure of $12, \mathrm{C}_{12} \mathrm{H}_{10} \mathrm{BrNO}$, was determined by single-crystal X-ray crystallography on a crystal isolated from methylene chloride/hexanes. The crystal selected was representative of the bulk sample. Crystal data at $100 \mathrm{~K}$ :

$$
\begin{array}{lll}
\mathrm{a}=8.7335(6) \AA & \alpha=90.00^{\circ} & \mathrm{V}=1076.98(13) \AA^{3} \\
\mathrm{~b}=9.1351(6) & \beta=90.00 & \text { Space group }=\mathrm{P} 2{ }_{1} 2_{1} 2_{1}, \# 19 \\
\mathrm{c}=13.4992(9) & \gamma=90.00 & \mathrm{Z}=4
\end{array}
$$

Data were collected on a Bruker CCD diffractometer using molybdenum Ka radiation and integrated to a resolution of $0.79 \AA^{-1}$ which yielded 2235 unique reflections from 11423 measured reflections.

The structure was solved using direct methods. The refined model has all non- $\mathrm{H}$ atoms refined anisotropically, and $\mathrm{H}$ atoms at their calculated positions, with agreement statistics of: $\mathrm{R}_{1}=$ $2.4 \%$, for 138 variables and 2208 reflections and $\mathrm{wR}_{2}=5.5 \%$ using all 2235 reflections. Based on this structure, the absolute configuration of the compound was determined to be $(R)$ (6-bromopyridin-2-yl)(phenyl)methanol.

The structure model is shown below and a perspective view calculated from the 
crystallographic coordinates is presented in Figure 1 and below.

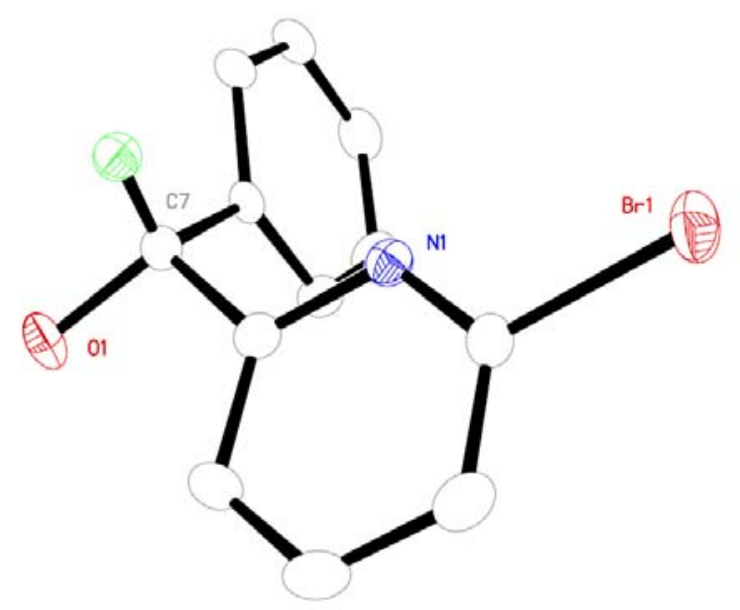

(6-bromopyridin-2-yl)(4-fluorophenyl)methanol (13). Prepared according to general procedure A from the corresponding ketone (4.0g, $14.2 \mathrm{mmol})$ and KRED124 to provide alcohol 13 (>99\%ee). The crude product was chromatographed with $15 \%$ EtOAc/Hexanes to provide the title compound as a white solid in $82 \%$ yield $(3.75 \mathrm{~g}),>99 \%$ ee. ${ }^{1} \mathrm{H}$ NMR (400 MHz, $\left.\mathrm{CDCl}_{3}\right) \delta 7.50(\mathrm{t}, J=7.7 \mathrm{~Hz}, 1 \mathrm{H}, \mathrm{pyH}), 7.41-7.33\left(\mathrm{~m}, 3 \mathrm{H}, \mathrm{C}_{6} \mathbf{H}_{4} \mathrm{~F}\right.$, pyH), $7.11(\mathrm{~d}, J=7.6 \mathrm{~Hz}, 1 \mathrm{H}, \mathrm{pyH}), 7.07-7.03\left(\mathrm{~m}, 2 \mathrm{H}, 5.76, \mathrm{C}_{6} \mathbf{H}_{4} \mathrm{~F}\right), 5.74(\mathrm{~s}, 1 \mathrm{H}$, PhCHOHpy); ${ }^{13} \mathrm{C}$ NMR (100 MHz, $\left.\mathrm{CDCl}_{3}\right) \delta 163.8,162.8,161.4,141.0,139.3,138.1,120.0$, 115.7, 115.5, 74.42; ${ }^{19} \mathrm{~F}$ NMR (400 MHz, $\mathrm{CDCl}_{3}$ ) $\delta$-114.6; HRMS (MALDI-TOF) exact mass calculated for $\left(\mathrm{C}_{12} \mathrm{H}_{10} \mathrm{BrFNO}\right)$ requires $\mathrm{m} / \mathrm{z} 281.99243$, found $\mathrm{m} / \mathrm{z} 281.99283(\mathrm{M}+\mathrm{H})^{+}$; $[\alpha]_{\mathrm{D}}=-10.7^{\circ}\left(\mathrm{c}=1.0, \mathrm{CHCl}_{3}\right)$; Melting point $53.0^{\circ} \mathrm{C}$ to $54.3^{\circ} \mathrm{C}$. Enantiomeric ratios were determined by SFC with a Chiralcel OF column $(250 \times 4.6 \mathrm{~mm} 10 \mu \mathrm{m} \mathrm{SFC,} \mathrm{MeOH} / \mathrm{CO} 2,1.5$ $\mathrm{mL} / \mathrm{min}, 35{ }^{\circ} \mathrm{C}, 200 \mathrm{bar}, 215 \mathrm{~nm}, 4 \%$ modifier for 4 min then $4 \%$ to $40 \%$ modifier at $2 \%$ per $\min ) ; \mathrm{t}_{\mathrm{r}}=10.9 \mathrm{~min}$ and $11.9 \mathrm{~min}$.

(6-bromopyridin-2-yl)(4-chlorophenyl)methanol (14). Prepared according to general procedure A from the corresponding ketone (1.0g, $3.4 \mathrm{mmol})$ and KRED24 to provide alcohol 14 ( $>99 \%$ ee). The crude product was chromatographed with $15 \%$ ethyl acetate/hexanes to provide the title compound as an oil in $79 \%$ yield $(0.80 \mathrm{~g}),>99 \%$ ee. ${ }^{1} \mathrm{H}$ NMR (400 MHz, $\left.\mathrm{CDCl}_{3}\right) \delta 7.48$ (t, $\left.J=7.7 \mathrm{~Hz}, 1 \mathrm{H}, \mathrm{pyH}\right), 7.38$ (d, $\left.J=7.8 \mathrm{~Hz}, 1 \mathrm{H}, \mathrm{pyH}\right), 7.33-$ 7.29 (m, 4H, $\mathrm{C}_{6} \mathbf{H}_{4} \mathrm{Cl}$ ), 7.12 (d, $\left.J=7.6 \mathrm{~Hz}, 1 \mathrm{H}, \mathrm{pyH}\right), 5.72$ (s, 1H, PhCHOHpy); ${ }^{13} \mathrm{C}$ NMR 
$\left(100 \mathrm{MHz}, \mathrm{CDCl}_{3}\right) \delta 162.6,141.0,140.8,139.4,134.0,128.9,128.4,127.1,120.0,74.4 ;$ HRMS (MALDI-TOF) exact mass calculated for $\left(\mathrm{C}_{12} \mathrm{H}_{10} \mathrm{BrClNO}\right)$ requires $\mathrm{m} / \mathrm{z} 297.96288$, found $\mathrm{m} / \mathrm{z} 297.96440(\mathrm{M}+\mathrm{H})^{+} ;[\alpha]_{\mathrm{D}}=-7.0^{\circ}\left(\mathrm{c}=1.0, \mathrm{CHCl}_{3}\right)$. Enantiomeric ratios were determined by SFC with a Chiralcel AD-H column $(250 \times 4.6 \mathrm{~mm} 10 \mu \mathrm{m} \mathrm{SFC}$, isocratic $15 \%$ $\left.\mathrm{MeOH} / \mathrm{CO}_{2}, 1.5 \mathrm{~mL} / \mathrm{min}, 35^{\circ} \mathrm{C}, 200 \mathrm{bar}, 215 \mathrm{~nm}, 15 \mathrm{~min}\right) ; \mathrm{t}_{\mathrm{r}}=9.3 \mathrm{~min}$ and $10.8 \mathrm{~min}$.

(6-bromopyridin-2-yl)(m-tolyl)methanol (15). Prepared according to general procedure A from the corresponding ketone $(10.0 \mathrm{~g}, 36 \mathrm{mmol})$ and KRED8 to provide alcohol 15 (70\%ee). The crude product was recrystallized from MTBE/hexanes. The mother liquors were concentrated and then purified by flash chromatography to provide the title compound as a white solid in $49 \%$ yield $(4.9 \mathrm{~g}), 83 \%$ ee. ${ }^{1} \mathrm{H}$ NMR $\left(400 \mathrm{MHz}, \mathrm{CDCl}_{3}\right) \delta 7.48(\mathrm{t}, J=7.7 \mathrm{~Hz}$, $1 \mathrm{H}$, pyH), $7.38(\mathrm{~d}, J=7.8 \mathrm{~Hz}, 1 \mathrm{H}, \mathrm{pyH}), 7.27-7.10\left(\mathrm{~m}, 5 \mathrm{H}, \mathrm{C}_{6} \mathbf{H}_{4}, \mathrm{pyH}\right), 5.72(\mathrm{~s}, 1 \mathrm{H}$, PhCHOHpy), 2.38 (s, 3H, PhCH $\left.\mathbf{H}_{3}\right) ;{ }^{13} \mathrm{C}$ NMR $\left(100 \mathrm{MHz}, \mathrm{CDCl}_{3}\right) \delta 163.3,142.4,141.0,139.3$, 138.6, 129.1, 128.8, 127.8, 126.9, 124.3, 120.3, 75.3, 21.6; HRMS (MALDI-TOF) exact mass calculated for $\left(\mathrm{C}_{13} \mathrm{H}_{12} \mathrm{BrNONa}\right)$ requires $\mathrm{m} / \mathrm{z} 299.99945$, found $\mathrm{m} / \mathrm{z} 299.99903(\mathrm{M}+\mathrm{Na})^{+} ;[\alpha]_{\mathrm{D}}$ $=-9.2^{\circ}\left(\mathrm{c}=1.0, \mathrm{CHCl}_{3}\right)$; Melting point $62.3{ }^{\circ} \mathrm{C}$ to $64.0{ }^{\circ} \mathrm{C}$. Enantiomeric ratios were determined by SFC with a Chiralcel AD-H column (250x4.6mm $10 \mu \mathrm{m} \mathrm{SFC}$, isocratic $15 \%$ $\left.\mathrm{MeOH} / \mathrm{CO}_{2}, 1.5 \mathrm{~mL} / \mathrm{min}, 35^{\circ} \mathrm{C}, 200 \mathrm{bar}, 215 \mathrm{~nm}, 15 \mathrm{~min}\right) ; \mathrm{t}_{\mathrm{r}}=7.1 \mathrm{~min}$ and $8.1 \mathrm{~min}$.

(6-bromopyridin-2-yl)(3-methoxyphenyl)methanol (16). Prepared according to general procedure A from the corresponding ketone $(1.0 \mathrm{~g}, 3.42 \mathrm{~g})$ to provide the desired alcohol ( $87 \%$ ee). The crude product was purified by flash chromatography with $20 \%$ ethyl acetate/hexanes to provide the title compound as a white solid in $76 \%$ yield $(0.76 \mathrm{~g}), 87 \%$ ee. ${ }^{1} \mathrm{H}$ NMR (400 MHz, CDCl $) \delta 7.49$ (t, $\left.J=7.7 \mathrm{~Hz}, 1 \mathrm{H}, \mathrm{pyH}\right), 7.39$ (d, $J=7.8 \mathrm{~Hz}, 1 \mathrm{H}$, pyH), 7.27 (t, $J=8.3 \mathrm{~Hz}, 1 \mathrm{H}, \mathrm{PhH}), 7.15(\mathrm{~d}, J=7.6 \mathrm{~Hz}, 1 \mathrm{H}, \mathrm{pyH}), 6.98-6.95\left(\mathrm{~m}, 2 \mathrm{H}, \mathrm{C}_{6} \mathbf{H}_{4}\right), 6.83$ (dd, $J=8.2,2.4,1 \mathrm{H}, \mathrm{C}_{6} \mathbf{H}_{4}$ ), 5.72 (s, $\left.1 \mathrm{H}, \mathrm{PhCHOHpy}\right), 3.80$ (s, $\left.3 \mathrm{H}, \mathrm{PhOCH}_{3}\right) ;{ }^{13} \mathrm{C}$ NMR (100 $\left.\mathrm{MHz}, \mathrm{CDCl}_{3}\right) \delta 162.9,160.0,143.8,140.9,139.3,129.8,126.9,120.1,119.4,113.7,113.5$, 75.0, 55.3; HRMS (MALDI-TOF) exact mass calculated for $\left(\mathrm{C}_{13} \mathrm{H}_{13} \mathrm{BrNO}_{2}\right)$ requires $\mathrm{m} / \mathrm{z}$ 294.01242, found $\mathrm{m} / \mathrm{z} 294.01216(\mathrm{M}+\mathrm{H})^{+} ;[\alpha]_{\mathrm{D}}=-17.3^{\circ}\left(\mathrm{c}=1.0, \mathrm{CHCl}_{3}\right)$; Melting point 68.1 ${ }^{\circ} \mathrm{C}$ to $69.0{ }^{\circ} \mathrm{C}$. Enantiomeric ratios were determined by SFC with a Chiralcel AD-H column 
(250x4.6mm $5 \mu \mathrm{m} \mathrm{SFC}$, isocratic $25 \% \mathrm{MeOH} / \mathrm{CO}_{2}, 1.5 \mathrm{~mL} / \mathrm{min}, 35^{\circ} \mathrm{C}$, 200bar, $215 \mathrm{~nm}, 15$ $\min ) ; \mathrm{t}_{\mathrm{r}}=5.8 \mathrm{~min}$ and $6.2 \mathrm{~min}$.

(6-methylpyridin-2-yl)(phenyl)methanol (20). Prepared according to general procedure A from the corresponding ketone $(10 \mathrm{~g}, 50.7 \mathrm{mmol})$ to provide the desired alcohol (92\%ee). The crude product was recrystallized from MTBE to provide the title compound as a white solid in 67\% yield $(6.7 \mathrm{~g}),>99 \%$ ee. ${ }^{1} \mathrm{H} \mathrm{NMR}\left(400 \mathrm{MHz}, \mathrm{CDCl}_{3}\right) \delta 7.50(\mathrm{t}, J=7.7 \mathrm{~Hz}$, 1H, pyH), 7.41-7.27 (m, 5H, PhH), 7.05 (d, $J=7.7 \mathrm{~Hz}, 1 \mathrm{H}$, pyH), 6.91 (d, $J=7.8 \mathrm{~Hz}, 1 \mathrm{H}$, pyH), 5.72 (s, 1H, PhCHOHpy), 2.60 (s, 3H, pyCH 3 ); ${ }^{13} \mathrm{C} \mathrm{NMR} \mathrm{(100} \mathrm{MHz,} \mathrm{CDCl}_{3}$ ) $\delta 159.9$, 156.7, 143.5, 137.2, 128.6, 127.8, 127.2, 121.9, 118.4, 74.6 24.3; HRMS (MALDI-TOF) exact mass calculated for $\left(\mathrm{C}_{13} \mathrm{H}_{14} \mathrm{NO}\right)$ requires $\mathrm{m} / \mathrm{z} 200.10699$, found $\mathrm{m} / \mathrm{z} 200.10793(\mathrm{M}+\mathrm{H})^{+}$; $[\alpha]_{\mathrm{D}}=+5.4^{\circ}\left(\mathrm{c}=1.0, \mathrm{CHCl}_{3}\right)$; melting point $112.5^{\circ} \mathrm{C}$ to $113.9^{\circ} \mathrm{C}$. Enantiomeric ratios were determined by SFC with a Chiralpak AD-H $(250 \times 4.6 \mathrm{~mm})$ column with a flow rate of 1.5 $\mathrm{mL} / \mathrm{min}\left(25 \% \mathrm{MeOH}\right.$ modifier, $\left.215 \mathrm{~nm}, 200 \mathrm{bar}, 35^{\circ} \mathrm{C}\right) ; \mathrm{t}_{\mathrm{r}}=16.6 \mathrm{~min}$ and $17.7 \mathrm{~min}$.

(6-chloropyridin-2-yl)(phenyl)methanol (21). Prepared according to general procedure A from the corresponding ketone $(1.0 \mathrm{~g}, 4.6 \mathrm{mmol})$ and KRED8 to provide the desired alcohol ( $83 \%$ ee). The crude product was purified by column chromatography with $15 \%$ ethyl acetate/hexanes to provide the title compound as a white solid in $88 \%$ yield $(0.88 \mathrm{~g}), 83 \%$ ee. ${ }^{1} \mathrm{H} \mathrm{NMR}\left(400 \mathrm{MHz}, \mathrm{CDCl}_{3}\right) \delta 7.59$ (t, $\left.J=7.8 \mathrm{~Hz}, 1 \mathrm{H}, \mathrm{pyH}\right), 7.41-7.30$ (m, 5H, PhH), 7.23 (d, $J=7.8 \mathrm{~Hz}, 1 \mathrm{H}, \mathrm{pyH}), 7.12(\mathrm{~d}, J=7.8 \mathrm{~Hz}, 1 \mathrm{H}$, pyH), $5.76(\mathrm{~s}, 1 \mathrm{H}$, PhCHOHpy); ${ }^{13} \mathrm{C}$ NMR (100 MHz, $\left.\mathrm{CDCl}_{3}\right) \delta 162.6,150.4,142.3,139.5,128.7,128.2,127.1$, 123.0, 119.7, 75.1; HRMS (MALDI-TOF) exact mass calculated for $\left(\mathrm{C}_{12} \mathrm{H}_{11} \mathrm{CINO}\right)$ requires $\mathrm{m} / \mathrm{z} 220.05237$, found $\mathrm{m} / \mathrm{z} 220.05261(\mathrm{M}+\mathrm{H})^{+} ;[\alpha]_{\mathrm{D}}=-14.2^{\circ}\left(\mathrm{c}=1.0, \mathrm{CHCl}_{3}\right)$; melting point $82.5^{\circ} \mathrm{C}$ to $84.1{ }^{\circ} \mathrm{C}$. Enantiomeric ratios were determined by SFC with a Chiralpak AD-H $(250 \times 4.6 \mathrm{~mm})$ column with a flow rate of $1.5 \mathrm{~mL} / \mathrm{min}(25 \% \mathrm{MeOH}$ modifier, $215 \mathrm{~nm}, 200$ bar, $\left.35^{\circ} \mathrm{C}\right) ; \mathrm{t}_{\mathrm{r}}=15.2 \mathrm{~min}$ and $15.8 \mathrm{~min}$.

(S)-(3-chlorophenyl)(phenyl)methanol (33). Prepared according to general procedure A from the corresponding ketone $(1.0 \mathrm{~g}, 4.8 \mathrm{mmol})$ and KRED108 to provide the desired alcohol (>99\% ee). The crude product was purified by flash chromatography with 
$10 \%$ ethyl acetate/hexanes to provide the title compound as a colorless oil in $81 \%$ yield $(0.81 \mathrm{~g}),>99 \%$ ee. All data corresponds to know data. ${ }^{2}[\alpha]_{\mathrm{D}}=-3.5^{\circ}\left(\mathrm{c}=1.0, \mathrm{CHCl}_{3}\right)$. Enantiomeric ratios were determined by SFC with a Chiralcel AD-H column $(250 \times 4.6 \mathrm{~mm} 5$ $\mu \mathrm{m} \mathrm{SFC}$, isocratic $\left.10 \% \mathrm{MeOH} / \mathrm{CO}_{2}, 1.5 \mathrm{~mL} / \mathrm{min}, 35^{\circ} \mathrm{C}, 200 \mathrm{bar}, 215 \mathrm{~nm}, 15 \mathrm{~min}\right) ; \mathrm{t}_{\mathrm{r}}=8.3 \mathrm{~min}$ and $8.9 \mathrm{~min}$.

(1S,2S)-(1,2-bis(6-bromopyridin-2-yl)-1,2-diphenylethane) (5). Prepared according to general procedure B from the alcohol 12 (528 mg, $2.0 \mathrm{mmol},>99 \% \mathrm{ee}$ ). Crude product was analyzed by HPLC to be a 86:14 diastereomeric mixture of $\mathrm{C}_{2}$ :meso isomers and chromatographed from $10 \%$ EtOAc/hexanes to provide the title compound as a white solid in $89 \%$ yield (440 mg); $>99 \%$ ee $\left(\mathrm{C}_{2}\right.$ symmetric isomer). ${ }^{1} \mathrm{H} \mathrm{NMR}\left(400 \mathrm{MHz}, \mathrm{CDCl}_{3}\right) \delta 7.34-$ $7.29(\mathrm{~m}, 8 \mathrm{H}, \mathrm{PhH}$ and pyH), $7.21(\mathrm{dd}, J=0.67,7.6 \mathrm{~Hz}, 2 \mathrm{H}, \mathrm{pyH}), 7.11(\mathrm{dt}, J=0.67,8.0 \mathrm{~Hz}$, 4H, pyH), 7.06-6.97 (m, 2H, PhH), 5.29 (s, 2H, PhCHpy); ${ }^{13} \mathrm{C}$ NMR (100 MHz, $\left.\mathrm{CDCl}_{3}\right) \delta$ $163.8,141.2,141.1,138.5,129.0,128.3,126.6,125.4,123.5,57.2$; HRMS (MALDI-TOF) exact mass calculated for $\left(\mathrm{C}_{24} \mathrm{H}_{19} \mathrm{~N}_{2} \mathrm{Br}_{2}\right)$ requires $\mathrm{m} / \mathrm{z}$ 492.99095, found $\mathrm{m} / \mathrm{z} 492.99203$ $(\mathrm{M}+\mathrm{H})^{+} ;[\alpha]_{\mathrm{D}}=+25.35^{\circ}\left(\mathrm{c}=1.0, \mathrm{CHCl}_{3}\right)$. Melting point $151.9^{\circ} \mathrm{C}$ to $153.0^{\circ} \mathrm{C}$. Enantiomeric ratios were determined by SFC with a Chiralcel AD-H column $(250 \times 4.6 \mathrm{~mm} 5 \mu \mathrm{m} \mathrm{SFC}$, isocratic $\left.8 \% \mathrm{MeOH} / \mathrm{CO}_{2}, 1.5 \mathrm{~mL} / \mathrm{min}, 35^{\circ} \mathrm{C}, 200 \mathrm{bar}, 215 \mathrm{~nm}, 15 \mathrm{~min}\right) ; \mathrm{t}_{\mathrm{r}}\left(C_{2}\right.$ symmetric $)=$ $8.5 \mathrm{~min}$ and $9.0 \mathrm{~min}, \mathrm{t}_{\mathrm{r}}($ meso $)=10.1 \mathrm{~min}$.

\section{Single-Crystal X-Ray Crystallography of 5}

The structure of $5, \mathrm{C}_{24} \mathrm{H}_{18} \mathrm{Br}_{2} \mathrm{~N}_{2}$, was determined by single-crystal X-ray crystallography on a crystal isolated from dichloromethane/hexanes vapor diffusion. The crystal selected was representative of the bulk sample. Crystal data at $100 \mathrm{~K}$ :

$$
\begin{aligned}
& a=9.0694(5) \AA \quad \alpha=90.00^{\circ} \quad V=2081.4(2) \AA^{3} \\
& b=11.7804(6) \quad \beta=90.00 \quad \text { Space group }=P 2{ }_{1} 2_{1} 2_{1}, \# 19 \\
& c=19.4812(11) \quad \gamma=90.00 \quad Z=4
\end{aligned}
$$

Data were collected on a Bruker CCD diffractometer using molybdenum $\mathrm{K} \alpha$ radiation and

2 (a) Wu, P-Y; Wu, H-L; Uang, B-J. J. Org. Chem. 2006, 71, 833. (b) Truppo, M.D.; Pollard, D.; Devine, P. Org. Lett. 2007, 9, 335. 
integrated to a resolution of $0.79 \AA^{-1}$ which yielded 4584 unique reflections from 23314 measured reflections.

The structure was solved using direct methods. The refined model has all non-H atoms refined anisotropically, and $\mathrm{H}$ atoms at their calculated positions, with agreement statistics of: $R_{1}=$ $2.0 \%$, for 253 variables and 4408 reflections and $w R_{2}=5.1 \%$ using all 4584 reflections. Based on this structure, the absolute configuration of the compound was determined to be (1S,2S)-(1,2-bis(6-bromopyridin-2-yl)-1,2-diphenylethane).

The structure model is shown below and a perspective view calculated from the crystallographic coordinates is presented in Figure 1 and below.

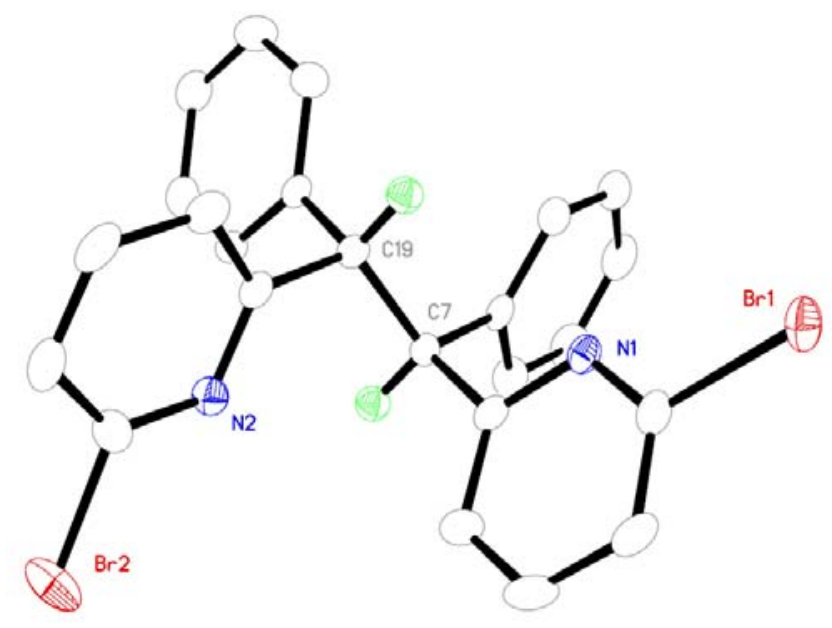

1,2-bis(6-bromopyridin-2-yl)-1,2-bis(4-fluorophenyl)ethane (22). Prepared according to general procedure B from alcohol $13(564 \mathrm{mg}, 2.0 \mathrm{mmol},>99 \%$ ee). The crude product was analyzed by HPLC to be a 73:27 diastereomeric mixture of $C_{2}$ :meso isomers and chromatographed from 5\% EtOAc/Hexanes to provide the title compound (inseparable mixture of diastereomers) as a white solid in $86 \%$ yield $(456 \mathrm{mg}) ;>99 \%$ ee $\left(C_{2}\right.$ symmetric isomer). $C_{2}$ symmetric isomer: ${ }^{1} \mathrm{H} \mathrm{NMR}\left(400 \mathrm{MHz}, \mathrm{CDCl}_{3}\right) \delta 7.31(\mathrm{t}, J=10.1 \mathrm{~Hz}, 2 \mathrm{H}$, pyH), 7.27 (dd, $J=8.9,5.4 \mathrm{~Hz}, 4 \mathrm{H}, \mathrm{PhH}), 7.15$ (d, $J=10.0 \mathrm{~Hz}, 2 \mathrm{H}, \mathrm{pyH}), 7.15$ (d, $J=10.0 \mathrm{~Hz}, 2 \mathrm{H}$, pyH), 6.83 (t, $J=8.9 \mathrm{~Hz}, 4 \mathrm{H}, \mathrm{PhH}), 5.06$ (s, 2H, PhCHрy); ${ }^{13} \mathrm{C}$ NMR (100 MHz, CDCl 3 ) $\delta$ 163.2, 141.2, 138.5, 136.6, 130.2, 125.5, 123.4, 115.1, 114.9, 56.5; HRMS (MALDI-TOF) exact mass calculated for $\left(\mathrm{C}_{24} \mathrm{H}_{17} \mathrm{~N}_{2} \mathrm{Br}_{2} \mathrm{~F}_{2}\right)$ requires $\mathrm{m} / \mathrm{z} 528.97211$, found $\mathrm{m} / \mathrm{z} 528.97418$ 
$(\mathrm{M}+\mathrm{H})^{+} ;[\alpha]_{\mathrm{D}}=+10.3^{\circ}\left(\mathrm{c}=1.0, \mathrm{CHCl}_{3}\right)$. Melting point $97^{\circ} \mathrm{C}$ to $98.5^{\circ} \mathrm{C}$. Enantiomeric ratios were determined by SFC with a Chiralcel OD-H column $(250 \times 4.6 \mathrm{~mm} 5 \mu \mathrm{m} \mathrm{SFC}$, IPA/CO $4 \%$ modifier for 4 min then ramp to $40 \%$ at $2 \%$ per minute and hold for 3 min at $40 \%$ IPA, $\left.1.5 \mathrm{~mL} / \mathrm{min}, 35^{\circ} \mathrm{C}, 200 \mathrm{bar}, 215 \mathrm{~nm}, 15 \mathrm{~min}\right) ; \mathrm{t}_{\mathrm{r}}\left(C_{2}\right.$ symmetric $)=11.9 \mathrm{~min}$ and $12.3 \mathrm{~min}, \mathrm{t}_{\mathrm{r}}$ $($ meso $)=13.2 \mathrm{~min}$.

\section{1,2-bis(6-bromopyridin-2-yl)-1,2-bis(4-chlorophenyl)ethane (23). Prepared} according to general procedure B from alcohol 14 (448 mg, $1.5 \mathrm{mmol},>99 \%$ ee). The crude product was analyzed by HPLC to be a 75:25 diastereomeric mixture of $C_{2}$ :meso isomers and chromatographed from $10 \%$ EtOAc/Hexanes to provide the title compound (inseparable mixture of diastereomers) as a white solid in $91 \%$ yield $(384 \mathrm{mg}) ;>99 \%$ ee $\left(C_{2}\right.$ symmetric isomer). $C_{2}$ symmetric and meso isomers (75:25 mixture): ${ }^{1} \mathrm{H} \mathrm{NMR}\left(400 \mathrm{MHz}, \mathrm{CDCl}_{3}\right) \delta 7.45$ (d, $J=8.5 \mathrm{~Hz}, 1 \mathrm{H}$, pyH), 7.32-7.23 (m, 5H, PhH, pyH), 7.17-7.10 (m, 7.5H, PhH, pyH), 6.98 (d, J =6.7 Hz, .5H, pyH), 5.07 (s, 1.5H, C 2 symmetric PhCHpy), 5.05 (s, .5H, meso PhCHpy); ${ }^{13} \mathrm{C}$ NMR $\left(100 \mathrm{MHz}, \mathrm{CDCl}_{3}\right) \delta 162.8,162.2,141.6,141.3,139.6,139.2,138.6,132.6,132.5$, 130.5, 128.5, 128.4, 125.8, 125.6, 123.4, 122.8, 56.9, 56.5; HRMS (MALDI-TOF) exact mass calculated for $\left(\mathrm{C}_{24} \mathrm{H}_{17} \mathrm{~N}_{2} \mathrm{Br}_{2} \mathrm{Cl}_{2}\right)$ requires $\mathrm{m} / \mathrm{z}$ 560.91301, found $\mathrm{m} / \mathrm{z} 560.01797(\mathrm{M}+\mathrm{H})^{+} ;[\alpha]_{\mathrm{D}}$ $=+13.5^{\circ}\left(\mathrm{c}=1.0, \mathrm{CHCl}_{3}\right)$. Melting point $151.0^{\circ} \mathrm{C}$ to $152.1{ }^{\circ} \mathrm{C}$. Enantiomeric ratios were determined by SFC with a Chiralpak OJ-H column (250x4.6mm $5 \mu \mathrm{m} \mathrm{SFC}$, isocratic $20 \%$ $\left.\mathrm{MeOH} / \mathrm{CO}_{2}, 1.5 \mathrm{~mL} / \mathrm{min}, 35^{\circ} \mathrm{C}, 200 \mathrm{bar}, 215 \mathrm{~nm}, 15 \mathrm{~min}\right) ; \mathrm{t}_{\mathrm{r}}\left(C_{2}\right.$ symmetric $)=7.11 \mathrm{~min}$ and $9.93 \mathrm{~min}, \mathrm{t}_{\mathrm{r}}($ meso $)=8.59 \mathrm{~min}$.

1,2-bis(6-bromopyridin-2-yl)-1,2-di-p-tolylethane (24). Prepared according to general procedure B from alcohol $15(556 \mathrm{mg}, 2.0 \mathrm{mmol}, 83 \% \mathrm{ee})$. The crude product was analyzed by NMR to be a $>95: 5$ diastereomeric mixture of $C_{2}$ :meso isomers and chromatographed from $5 \%$ EtOAc/Hexanes to provide the title compound (inseparable mixture of diastereomers) as a white solid in $89 \%$ yield $(464 \mathrm{mg}) ; 44 \%$ ee $\left(C_{2}\right.$ symmetric isomer). $C_{2}$ symmetric isomer: ${ }^{1} \mathrm{H} \mathrm{NMR}\left(400 \mathrm{MHz}, \mathrm{CDCl}_{3}\right) \delta 7.30(\mathrm{t}, J=7.6 \mathrm{~Hz}, 2 \mathrm{H}, \mathrm{pyH})$, 7.20 (d, $J=7.6 \mathrm{~Hz}, 2 \mathrm{H}, \mathrm{pyH}), 7.12-7.10$ (m, 6H, pyH, $\mathrm{PhH}), 7.11$ (t, $J=7.6 \mathrm{~Hz}, 2 \mathrm{H}, \mathrm{PhH}$ ),

6.86 (d, $J=7.5 \mathrm{~Hz}, 2 \mathrm{H}, \mathrm{PhH}), 5.10$ (s, 2H, PhCHpy), 2.21 (s, 6H, PhMe); ${ }^{13} \mathrm{C}$ NMR (100 $\left.\mathrm{MHz}, \mathrm{CDCl}_{3}\right) \delta 164.0,141.0,140.9,138.4,137.5,129.7,129.0,127.2,125.9,125.2,123.4$, 
57.0, 21.4; HRMS (MALDI-TOF) exact mass calculated for $\left(\mathrm{C}_{26} \mathrm{H}_{23} \mathrm{~N}_{2} \mathrm{Br}_{2}\right)$ requires $\mathrm{m} / \mathrm{z}$ 521.02225, found $\mathrm{m} / \mathrm{z} 521.02345(\mathrm{M}+\mathrm{H})^{+} ;[\alpha]_{\mathrm{D}}=+18.3^{\circ}\left(\mathrm{c}=1.0, \mathrm{CHCl}_{3}\right)$. Melting point $181.0^{\circ} \mathrm{C}$ to $182.1{ }^{\circ} \mathrm{C}$. Enantiomeric ratios were determined by SFC with a Chiralcel OJ-H (250x4.6mm $5 \mu \mathrm{m} \mathrm{SFC,} \mathrm{isocratic} \mathrm{15 \%} \mathrm{MeOH} / \mathrm{CO}_{2}, 1.5 \mathrm{~mL} / \mathrm{min}, 35^{\circ} \mathrm{C}, 200 \mathrm{bar}, 215 \mathrm{~nm}, 10$ $\min ) ; \mathrm{t}_{\mathrm{r}}\left(C_{2}\right.$ symmetric $)=5.8 \mathrm{~min}$ and $7.9 \mathrm{~min}$.

1,2-bis(6-bromopyridin-2-yl)-1,2-bis(4-methoxyphenyl)ethane (25). Prepared according to general procedure B from alcohol 16 (441 mg, $1.5 \mathrm{mmol}, 87 \%$ ee). Crude reaction mixture was analyzed by HPLC to be a 86:14 diastereomeric mixture of $C_{2}: m e s o$ isomers and chromatographed from EtOAc/Hexanes to provide the title compound (inseparable mixture of diastereomers) as a white solid in $80 \%$ yield $(332 \mathrm{mg}) ; 64 \%$ ee $\left(C_{2}\right.$ symmetric isomer). $C_{2}$ symmetric isomer: ${ }^{1} \mathrm{H} \mathrm{NMR}\left(400 \mathrm{MHz}, \mathrm{CDCl}_{3}\right) \delta 7.30(\mathrm{t}, J=7.6 \mathrm{~Hz}$, 2H, pyH), 7.27 (d, $J=7.5 \mathrm{~Hz}, 2 \mathrm{H}$, pyH), 7.21 (d, $J=7.6 \mathrm{~Hz}, 2 \mathrm{H}$, pyH), 7.12 (d, J=7.7 Hz, 2H, PhH), 7.04 (t, J = 7.5 Hz, 2H, PhH), 6.92-6.90 (m, 4H, PhH), 6.60 (d, J =7.5 Hz, 2H, $\mathrm{PhH}), 5.10$ (s, 2H, PhCHpy), 3.70 (s, 6H, PhOMe); ${ }^{13} \mathrm{C} \mathrm{NMR}\left(100 \mathrm{MHz}, \mathrm{CDCl}_{3}\right) \delta$ 163.6, $159.4,142.5,141.1,138.4,129.1,125.3,123.4,121.4,114.4,112.4,57.1,55.2$; HRMS (MALDI-TOF) exact mass calculated for $\left(\mathrm{C}_{26} \mathrm{H}_{23} \mathrm{~N}_{2} \mathrm{Br}_{2} \mathrm{O}_{2}\right)$ requires $\mathrm{m} / \mathrm{z} 553.01208$, found $\mathrm{m} / \mathrm{z}$ $553.01377(\mathrm{M}+\mathrm{H})^{+} ;[\alpha]_{\mathrm{D}}=+10.7^{\circ}\left(\mathrm{c}=1.0, \mathrm{CHCl}_{3}\right)$. Melting point $152.4^{\circ} \mathrm{C}$ to $152.8^{\circ} \mathrm{C}$. Enantiomeric ratios were determined by SFC with a Chiralcel AD-H $(250 x 4.6 \mathrm{~mm} 5 \mu \mathrm{m}$ SFC, isocratic $\left.15 \% \mathrm{MeOH} / \mathrm{CO}_{2}, 1.5 \mathrm{~mL} / \mathrm{min}, 35^{\circ} \mathrm{C}, 200 \mathrm{bar}, 215 \mathrm{~nm}, 10 \mathrm{~min}\right) ; \mathrm{t}_{\mathrm{r}}\left(C_{2}\right.$ symmetric $)=$ $4.7 \mathrm{~min}$ and $5.8 \mathrm{~min}$.

1,2-bis(6-methylpyridin-2-yl)-1,2-diphenylethane (29). Prepared according to general procedure B from the corresponding alcohol 20 (398 mg, $2.0 \mathrm{mmol},>99 \%$ ee). The crude product was analyzed by HPLC to be a 72:28 diastereomeric mixture of $C_{2}$ :meso isomers and chromatographed from EtOAc/Hexanes to provide the title compound (inseparable mixture of diastereomers) as a white solid in $51 \%$ yield $(186 \mathrm{mg}) ; 50 \%$ ee $\left(C_{2}\right.$ symmetric isomer). $C_{2}$ symmetric and meso isomers: ${ }^{1} \mathrm{H} \mathrm{NMR}\left(400 \mathrm{MHz}, \mathrm{CDCl}_{3}\right) \delta 7.59(\mathrm{~d}, J$ $=7.44 \mathrm{~Hz}, 1 \mathrm{H}, \mathrm{pyH}), 7.48$ (d, $J=7.35 \mathrm{~Hz}, 3 \mathrm{H}, \mathrm{pyH}), 7.35$ (m, 2H, PhH), 7.12-6.95 (m, 8H, $\mathrm{PhH}$ and pyH), 6.75 (d, $J=7.5 \mathrm{~Hz}, 2 \mathrm{H}, \mathrm{pyH}), 5.19$ (s, 2H, PhCHpy), 2.47 and 2.43 (s, 6H, pyMe); ${ }^{13} \mathrm{C}$ NMR $\left(100 \mathrm{MHz}, \mathrm{CDCl}_{3}\right) \delta 161.8,157.1,129.0,128.9,127.9,127.8,126.0,120.8$, 
120.4, 120.2, 109.6, 58.0, 24.5; HRMS (MALDI-TOF) exact mass calculated for $\left(\mathrm{C}_{26} \mathrm{H}_{25} \mathrm{~N}_{2}\right)$ requires $\mathrm{m} / \mathrm{z}$ 365.20123, found $\mathrm{m} / \mathrm{z} 365.20244(\mathrm{M}+\mathrm{H})^{+} ;[\alpha]_{\mathrm{D}}=-4.3^{\circ}\left(\mathrm{c}=1.0, \mathrm{CHCl}_{3}\right)$. Melting point $156.4{ }^{\circ} \mathrm{C}$ to $158.0{ }^{\circ} \mathrm{C}$. Enantiomeric ratios were determined by SFC with a Sepapak-2 (250x4.6mm $5 \mu \mathrm{m} \mathrm{SFC,} 4 \% \mathrm{MeOH} / \mathrm{CO}_{2}$ for $4 \mathrm{~min}$ then to $40 \%$ for $3 \mathrm{~min}, 1.5$ $\mathrm{mL} / \mathrm{min}, 35{ }^{\circ} \mathrm{C}, 200$ bar, $\left.215 \mathrm{~nm}, 25 \mathrm{~min}\right) ; \mathrm{t}_{\mathrm{r}}\left(C_{2}\right.$ symmetric $)=10.3 \mathrm{~min}$ and $10.7 \mathrm{~min}, \mathrm{t}_{\mathrm{r}}$ $($ meso $)=10.4 \mathrm{~min}$.

1,2-bis(6-chloropyridin-2-yl)-1,2-diphenylethane (30). Prepared according to general procedure B from the corresponding alcohol 21 (438mg, $2.0 \mathrm{mmol}, 83 \%$ ee), Crude reaction mixture was analyzed by HPLC to be a 85:15 diastereomeric mixture of $C_{2}$ :meso isomers and chromatographed from EtOAc/Hexanes to provide the title compound (inseparable mixture of diastereomers) as a white solid in 90\% yield (364 mg); 83\% ee $\left(C_{2}\right.$ symmetric isomer). $C_{2}$ symmetric isomer: ${ }^{1} \mathrm{H}$ NMR $\left(400 \mathrm{MHz}, \mathrm{CDCl}_{3}\right) \delta 7.39(\mathrm{t}, J=7.7 \mathrm{~Hz}$, 2H, pyH), 7.32 (d, J=7.6 Hz, 4H, PhH), 7.19 (d, J=7.6 Hz, 4H, PhH), 7.09 (t, J=7.6 Hz, 2H, PhH), 7.05 (d, J = $7.7 \mathrm{~Hz}, 2 \mathrm{H}$, pyH), 6.95 (d, J =7.8 Hz, 2H, pyH), 5.18 (s, 2H, PhCHрy); ${ }^{13} \mathrm{C}$ NMR (100 MHz, $\left.\mathrm{CDCl}_{3}\right) \delta 163.3,150.3,141.0,138.7,128.9,128.2,126.5$, 123.1, 121.5, 57.1; HRMS (MALDI-TOF) exact mass calculated for $\left(\mathrm{C}_{24} \mathrm{H}_{19} \mathrm{~N}_{2} \mathrm{Cl}_{2}\right)$ requires $\mathrm{m} / \mathrm{z} 405.09198$, found $\mathrm{m} / \mathrm{z} 405.09308(\mathrm{M}+\mathrm{H})^{+} ;[\alpha]_{\mathrm{D}}=+18.6^{\circ}\left(\mathrm{c}=1.0, \mathrm{CHCl}_{3}\right)$. Melting point $157.8{ }^{\circ} \mathrm{C}$ to $159.51{ }^{\circ} \mathrm{C}$. Enantiomeric ratios were determined by SFC with a tandem Chiralpak AS-H and Chiralpak OJ-H column $(250 \times 4.6 \mathrm{~mm} 5 \mu \mathrm{m}$ SFC, isocratic $8 \%$ $\left.\mathrm{MeOH} / \mathrm{CO}_{2}, 1.5 \mathrm{~mL} / \mathrm{min}, 35^{\circ} \mathrm{C}, 200 \mathrm{bar}, 215 \mathrm{~nm}, 25 \mathrm{~min}\right) ; \mathrm{t}_{\mathrm{r}}\left(C_{2}\right.$ symmetric $)=16.2 \mathrm{~min}$ and $18.0 \min , \mathrm{t}_{\mathrm{r}}($ meso $)=17.0 \mathrm{~min}$.

1,1,2,2-tetraphenylethane (32). Prepared according to general procedure B from benzhydrol (500 mg, $2.7 \mathrm{mmol})$ and $100 \mathrm{~mol} \% \mathrm{CuCN}(2.7 \mathrm{mmol})$ and aging reaction overnight. Crude reaction mixture chromatographed using EtOAc/Hexanes to provide the title compound as a white solid in $30 \%$ yield $(135 \mathrm{mg})$. Spectral data is consistent with that previously reported in the literature for this compound. ${ }^{3}$

\footnotetext{
${ }^{3}$ Inaba, Shin-ichi; Matsumoto, Hideyuki; Rieke, Reuben D. J. Org. Chem. 1984, 49, 2093-2098.
} 
1,2-bis(3-chlorophenyl)-1,2-diphenylethane (34). Prepared according to general procedure B from the corresponding alcohol 33 (436mg, $2.0 \mathrm{mmol}$, >99\% ee) and $10 \mathrm{~mol} \%$ $\mathrm{CuCN}$. Crude reaction mixture was analyzed by HPLC to be a 64:36 diastereomeric mixture of $C_{2}$ :meso isomers and chromatographed from EtOAc/Hexanes to provide the title compound (inseparable mixture of diastereomers) as a white solid in $54 \%$ yield $(218 \mathrm{mg}) ; 93 \%$ ee $\left(C_{2}\right.$ symmetric isomer). $C_{2}$ symmetric and meso isomers: ${ }^{1} \mathrm{H}$ NMR $\left(400 \mathrm{MHz}, \mathrm{CDCl}_{3}\right) \delta$ 7.17-7.14 (m, 10H, PhH), 7.08-7.02 (m, 8H, PhH), 4.71 (s, 2H, PhCHPh); ${ }^{13} \mathrm{C}$ NMR (100 MHz, $\mathrm{CDCl}_{3}$ ) $\delta 145.2,142.2,134.1,134.0,129.6,129.5,128.7,128.7,128.5,128.4,126.6,126.5,126.5$, 126.4, 126.3, 56.0; HRMS (MALDI-TOF) exact mass calculated for $\left(\mathrm{C}_{26} \mathrm{H}_{21} \mathrm{Cl}_{2}\right)$ requires $\mathrm{m} / \mathrm{z}$ 403.10148, found $\mathrm{m} / \mathrm{z} 403.10203(\mathrm{M}+\mathrm{H})^{+} ;[\alpha]_{\mathrm{D}}=-11.0^{\circ}\left(\mathrm{c}=1.0, \mathrm{CHCl}_{3}\right)$. Melting point $178.9{ }^{\circ} \mathrm{C}$ to $181.1^{\circ} \mathrm{C}$. Enantiomeric ratios were determined by SFC with a tandem Chiralpak OJ-H and Chiralpak OJ-H column $\left(250 \times 4.6 \mathrm{~mm} 5 \mu \mathrm{m} \mathrm{SFC}\right.$, isocratic $8 \% \mathrm{MeOH} / \mathrm{CO}_{2}, 1.5$ $\left.\mathrm{mL} / \mathrm{min}, 30^{\circ} \mathrm{C}, 200 \mathrm{bar}, 215 \mathrm{~nm}, 35 \mathrm{~min}\right) ; \mathrm{t}_{\mathrm{r}}\left(C_{2}\right.$ symmetric $)=28.0 \mathrm{~min}$ and $30.5 \mathrm{~min}, \mathrm{t}_{\mathrm{r}}($ meso $)$ $=29.4 \mathrm{~min}$. 


\section{Spectral and Crystallographic Data}

Spectra for Alcohol 12
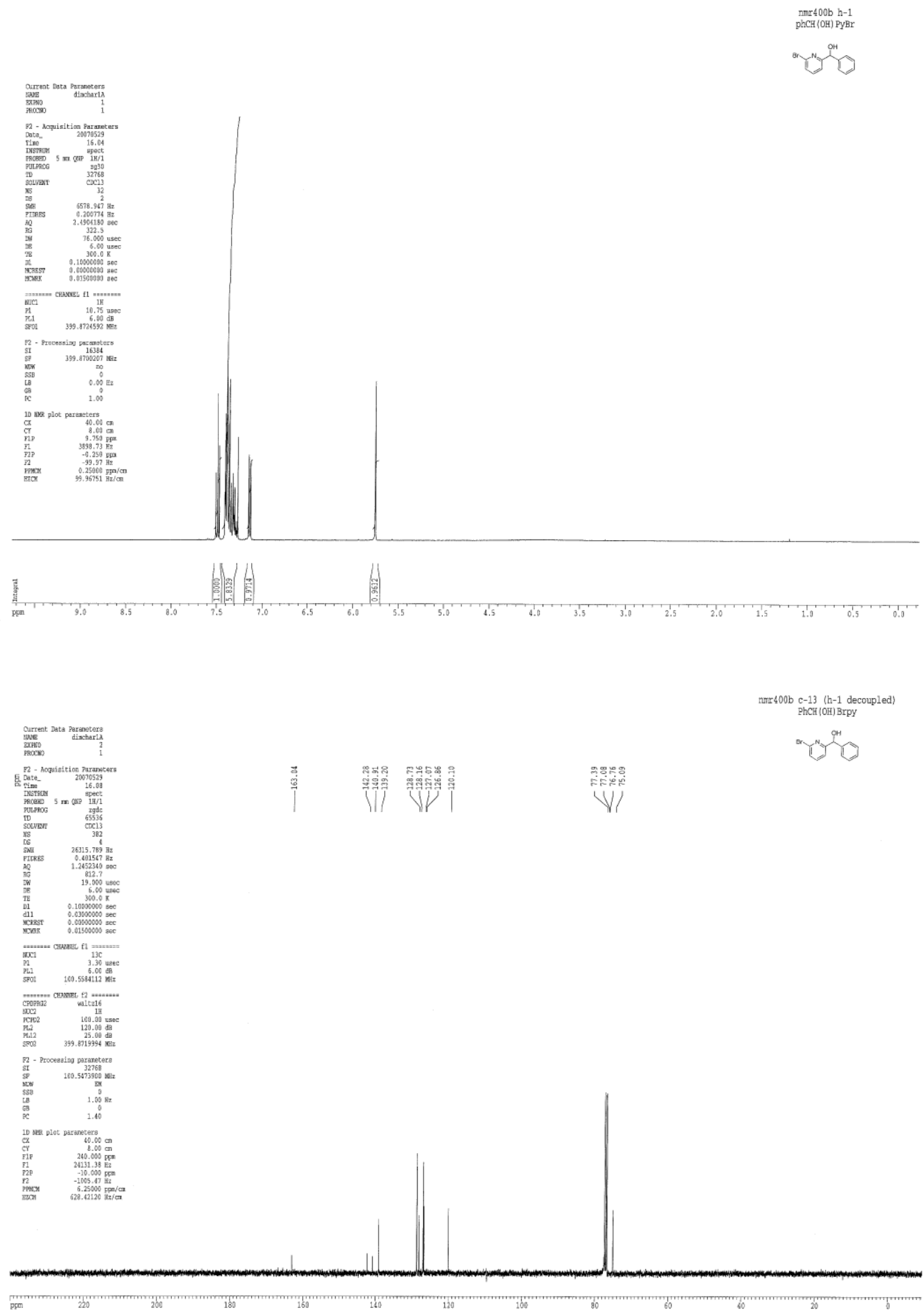
Crystal structure data: Compound $\mathrm{C}_{12} \mathrm{H}_{10} \mathrm{Br} \mathrm{N}$ O, $M_{r}=$ 264.120, orthorhombic, $P 2{ }_{1} 2_{1} 2_{1}, a=8.7335(6), b=9.1351(6), c$ $=13.4992(9) \AA, V=1076.98(13) \AA^{3}, Z=4, D_{x}=1.629 \mathrm{gcm}^{-3}$, monochromatized radiation $\lambda(\mathrm{Mo})=0.71073 \AA, \mu=3.79 \mathrm{~mm}^{-1}$, $F(000)=528, T=100^{\circ} \mathrm{K}$. Data were collected on a Bruker

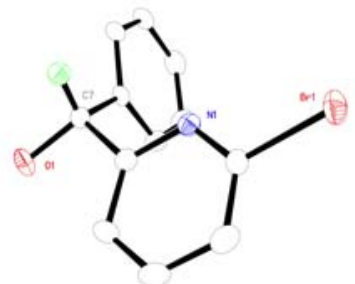
CCD diffractometer to a $\theta$ limit of $26.73^{\circ}$ which yielded 11423 reflections. There are 2235 unique reflections with 2208 observed at the $2 \sigma$ level. The structure was solved by direct methods (SHELXS-97, Sheldrick, G.M. Acta Crystallogr., 1990, A46, 467-473) and refined using full-matrix least-squares on $F^{2}$ (SHELXL-97, Sheldrick, G.M. SHELXL-97. Program for the Refinement of Crystal Structures. Univ. of Göttingen, Germany). The final model was refined using 138 parameters and all 2235 data. All non-hydrogen atoms were refined with anisotropic thermal displacements. The final agreement statistics are: $R=0.024$ (based on 2208 reflections with $I>2 \sigma(I)), w R=0.055, S=1.07$ with $(\Delta / \sigma)_{\max }<0.01$. The maximum peak height in a final difference Fourier map is $0.456 \mathrm{e} \AA^{-3}$ and this peak is without chemical significance. CCDC 667023 contains the supplementary crystallographic data for this paper. These data can be obtained free of charge from The Cambridge Crystallographic Data Centre via www.ccdc.cam.ac.uk/data_request/cif. 
Spectra for Alcohol 13
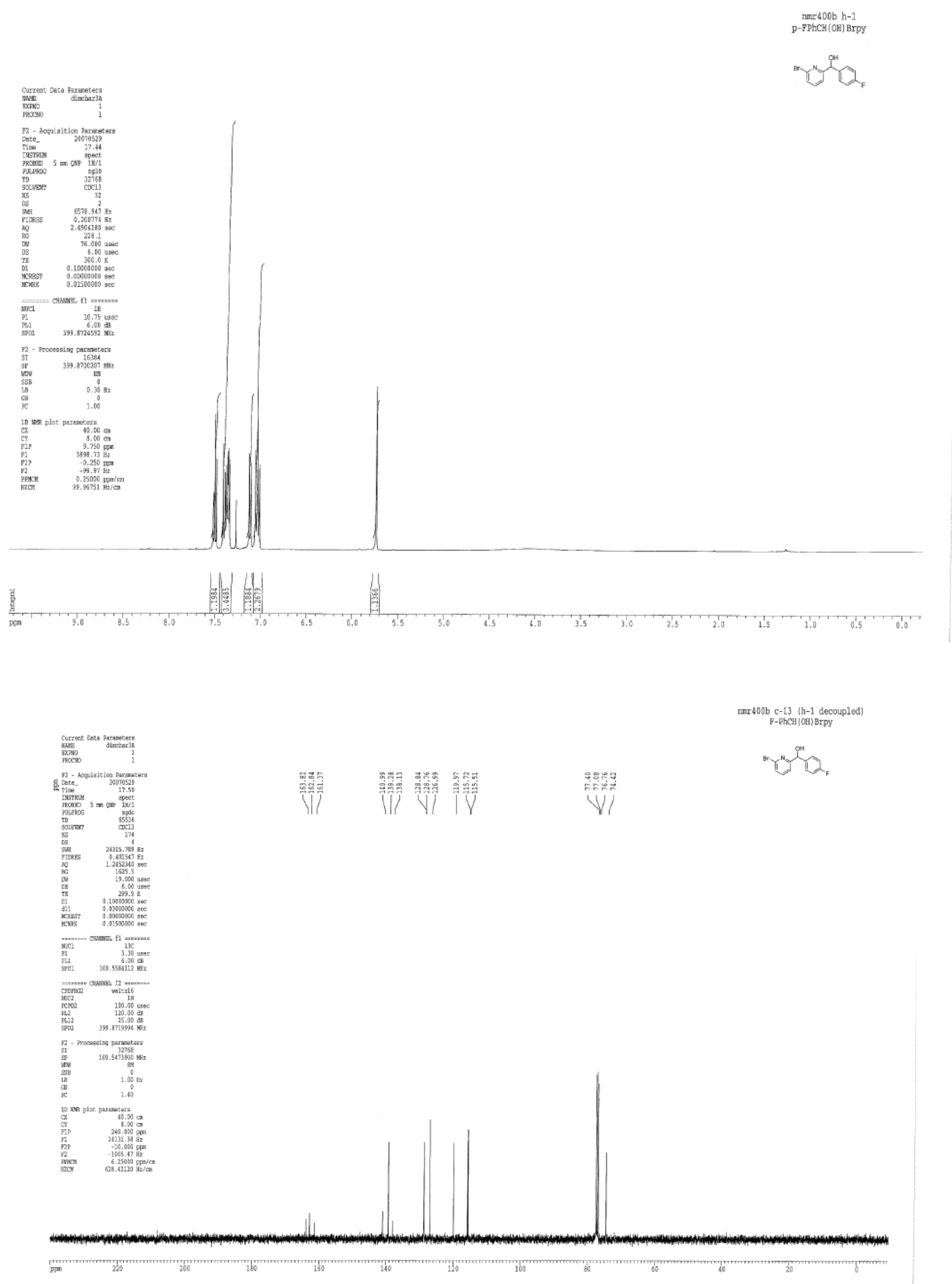


\section{Spectra for Alcohol 14}

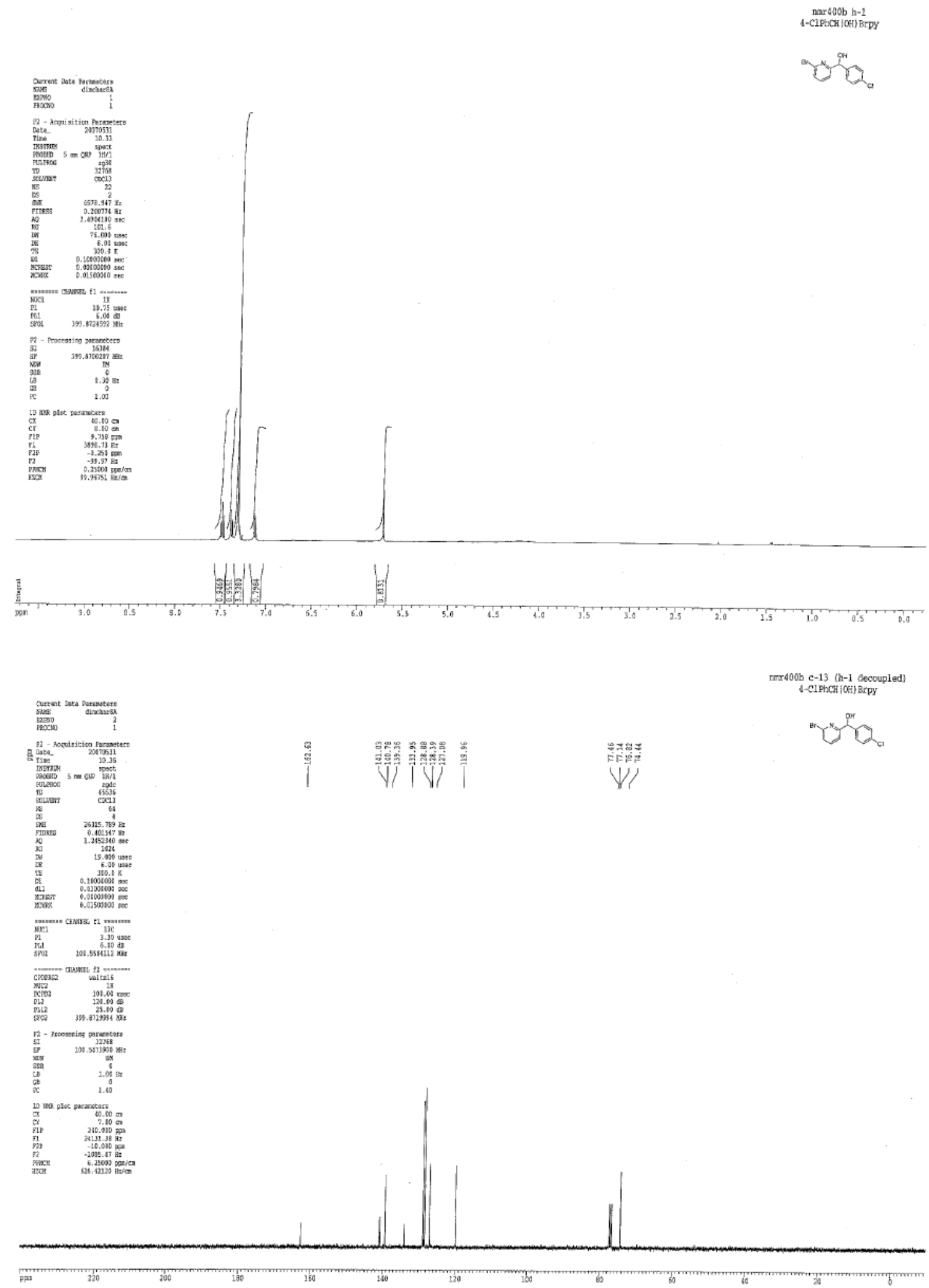


Spectra for Alcohol 15

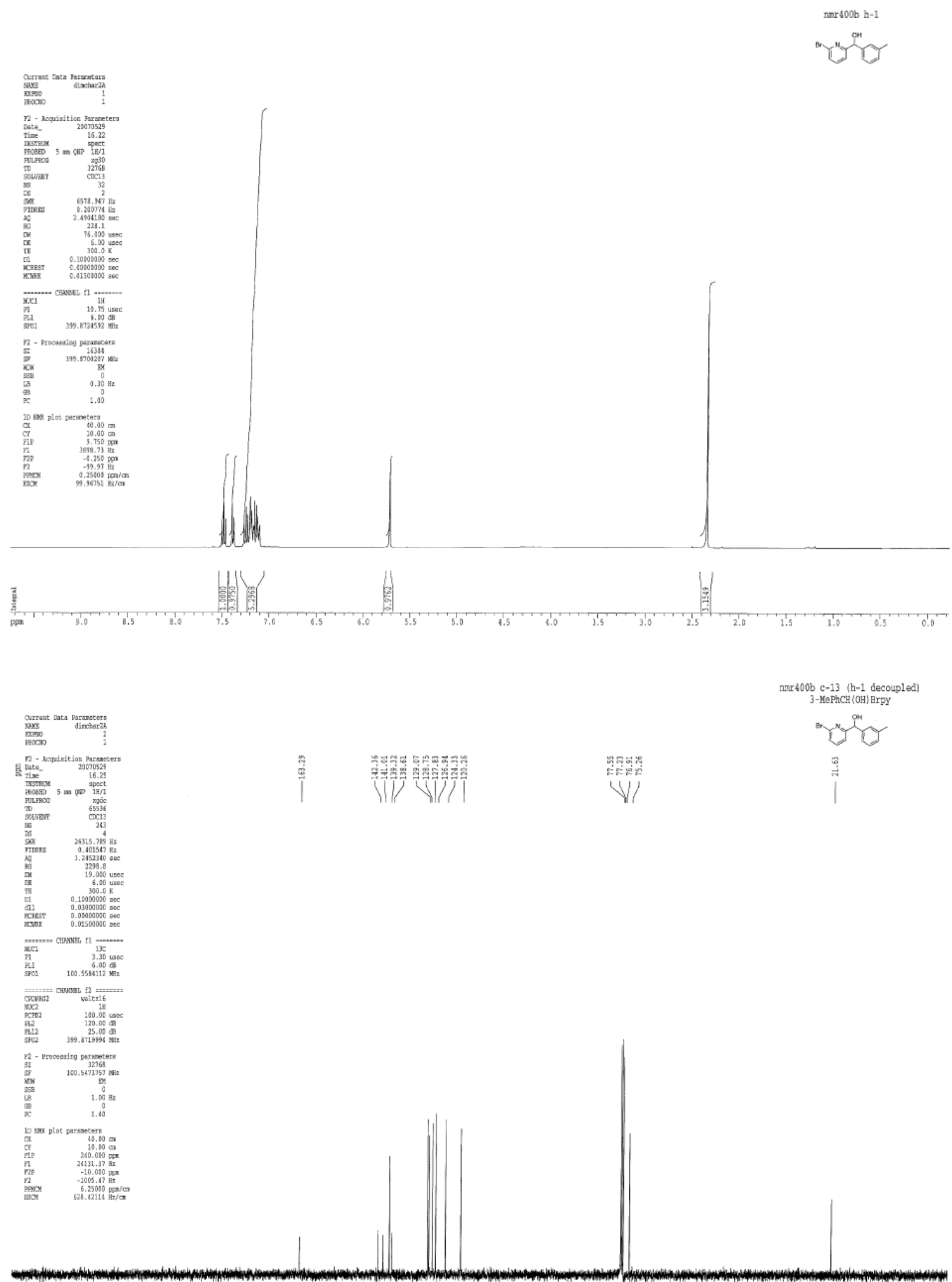

ivin

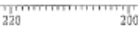

${ }_{200}$

${ }_{160}$

${ }_{120}$

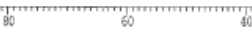


Spectra for Alcohol 16

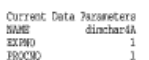

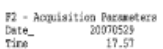

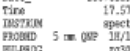

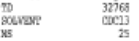

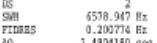

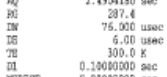

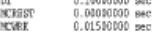

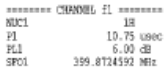

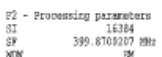

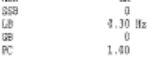

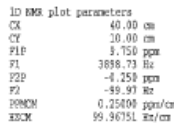

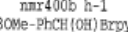

I

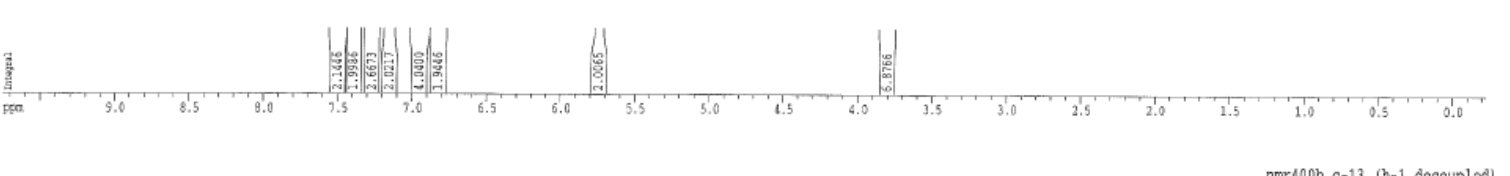

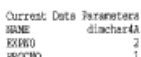

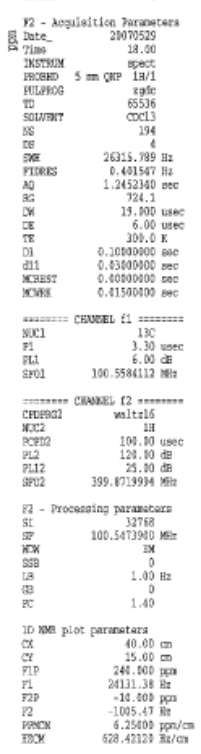

ㅇํำ

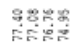

VI

"Conc 
Spectral Data for 20
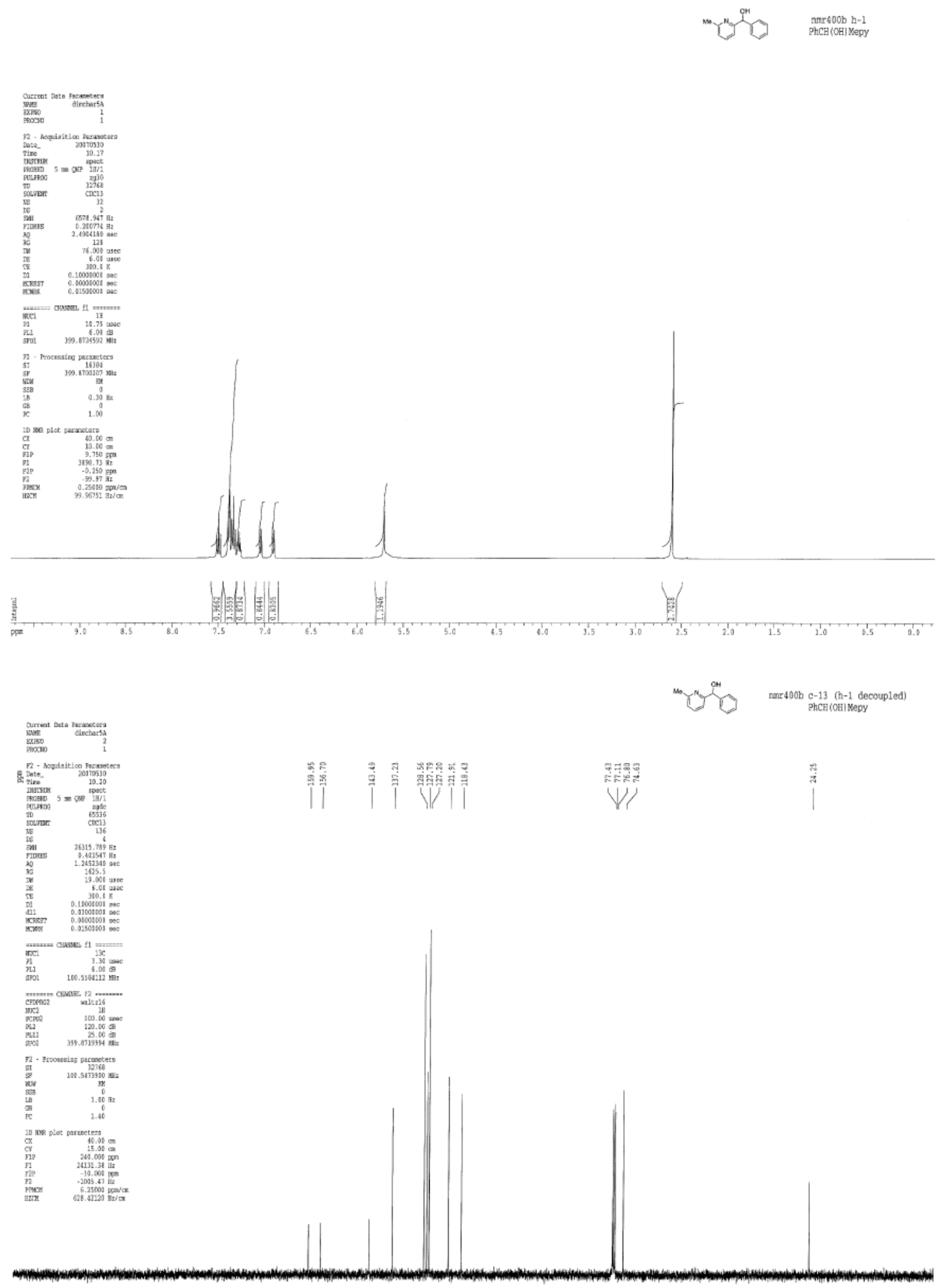

(hnr $400 \mathrm{~b}$ c-13 (h-1 decoupled)
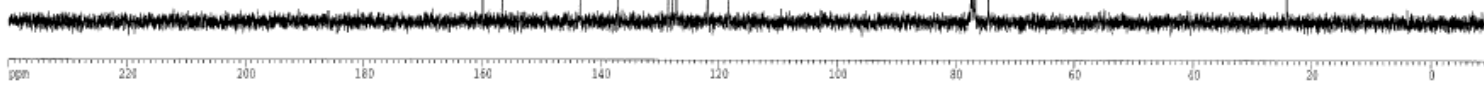


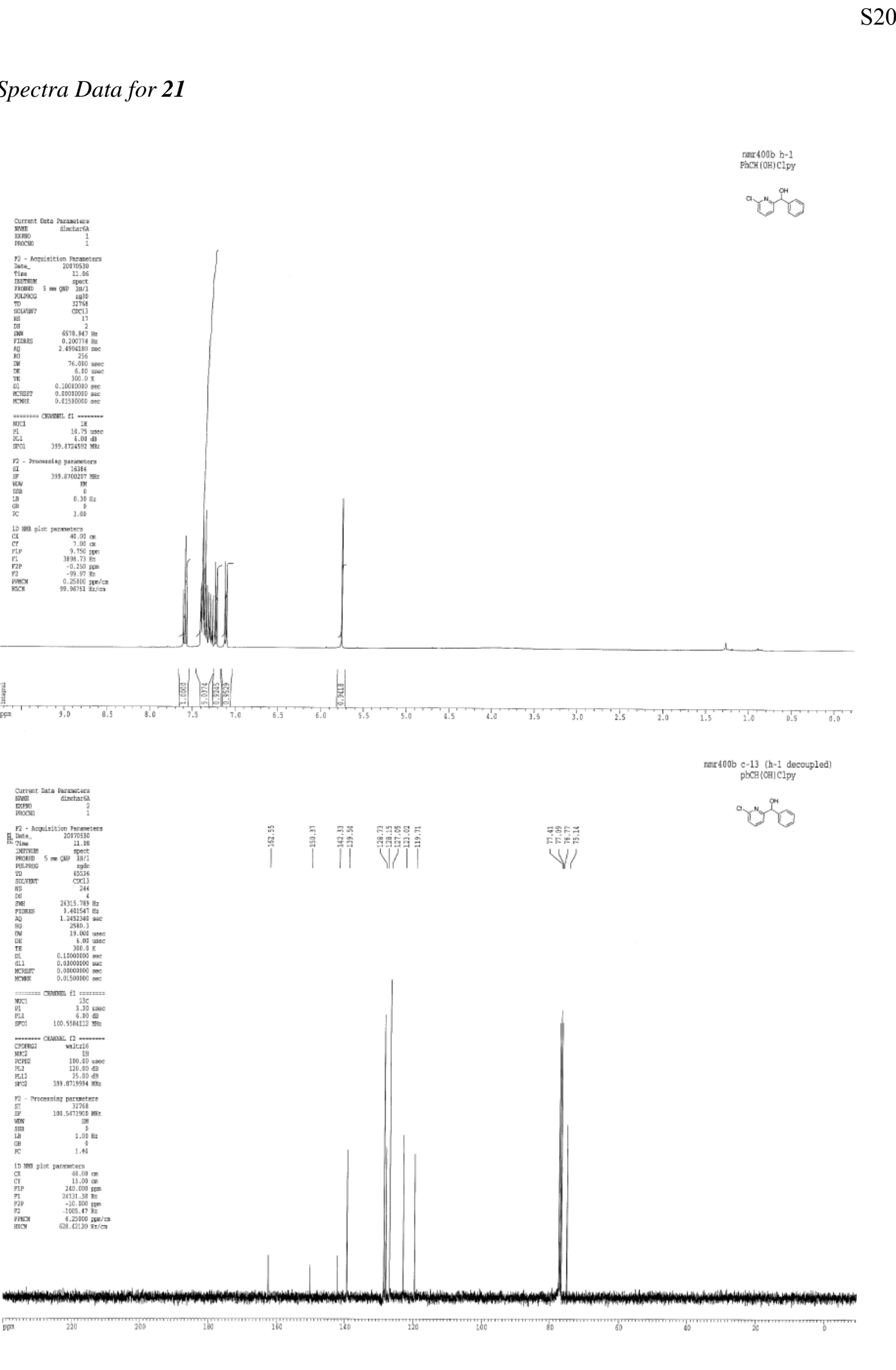

nanr $400 \mathrm{~b}$ h-1
PhCH (OOH)Clpy

aris
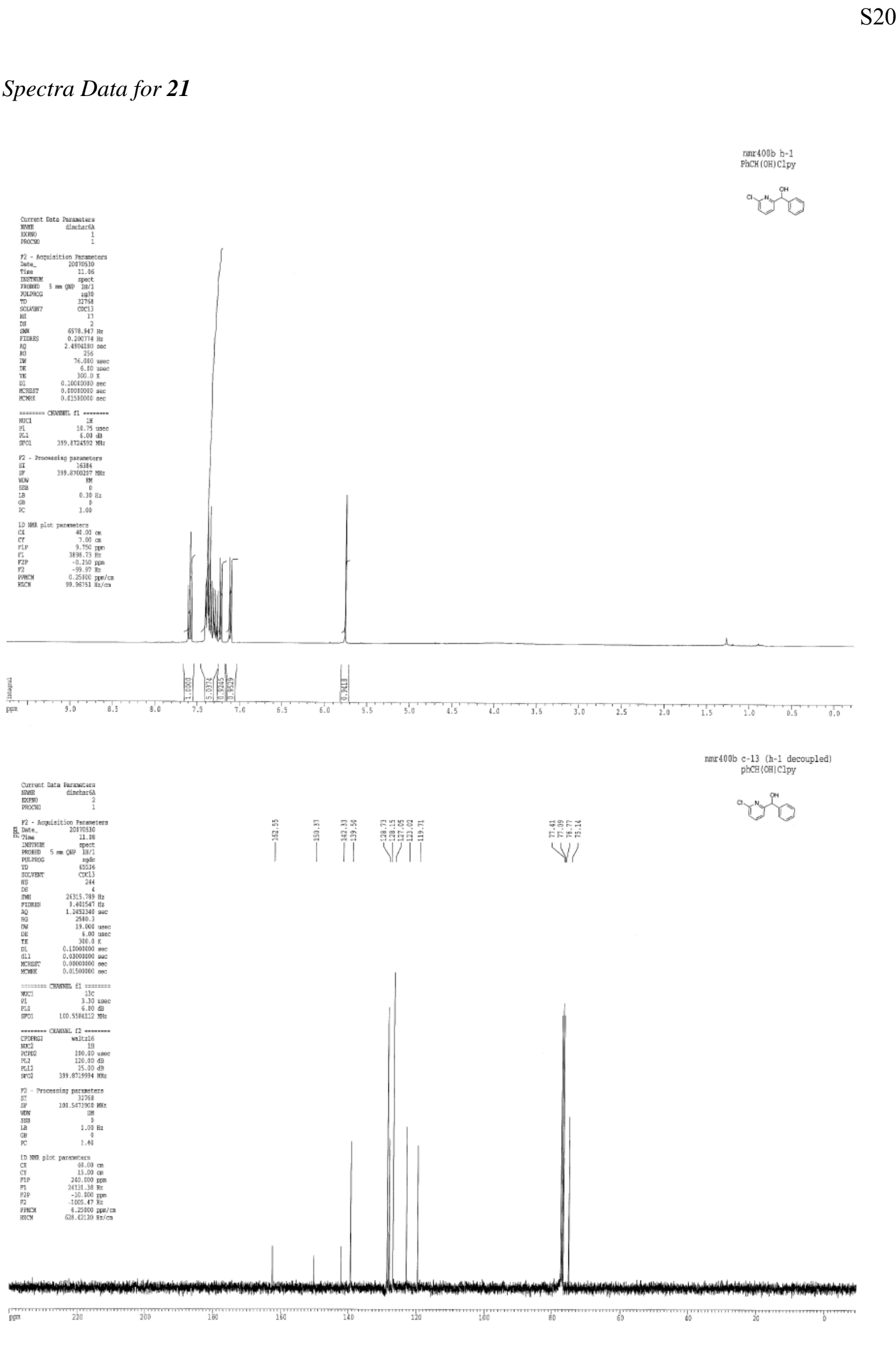

$$
\text { s, }
$$

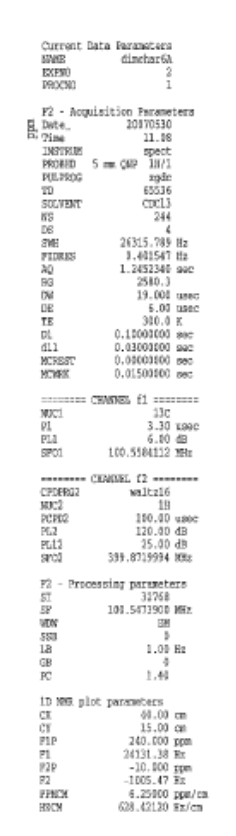
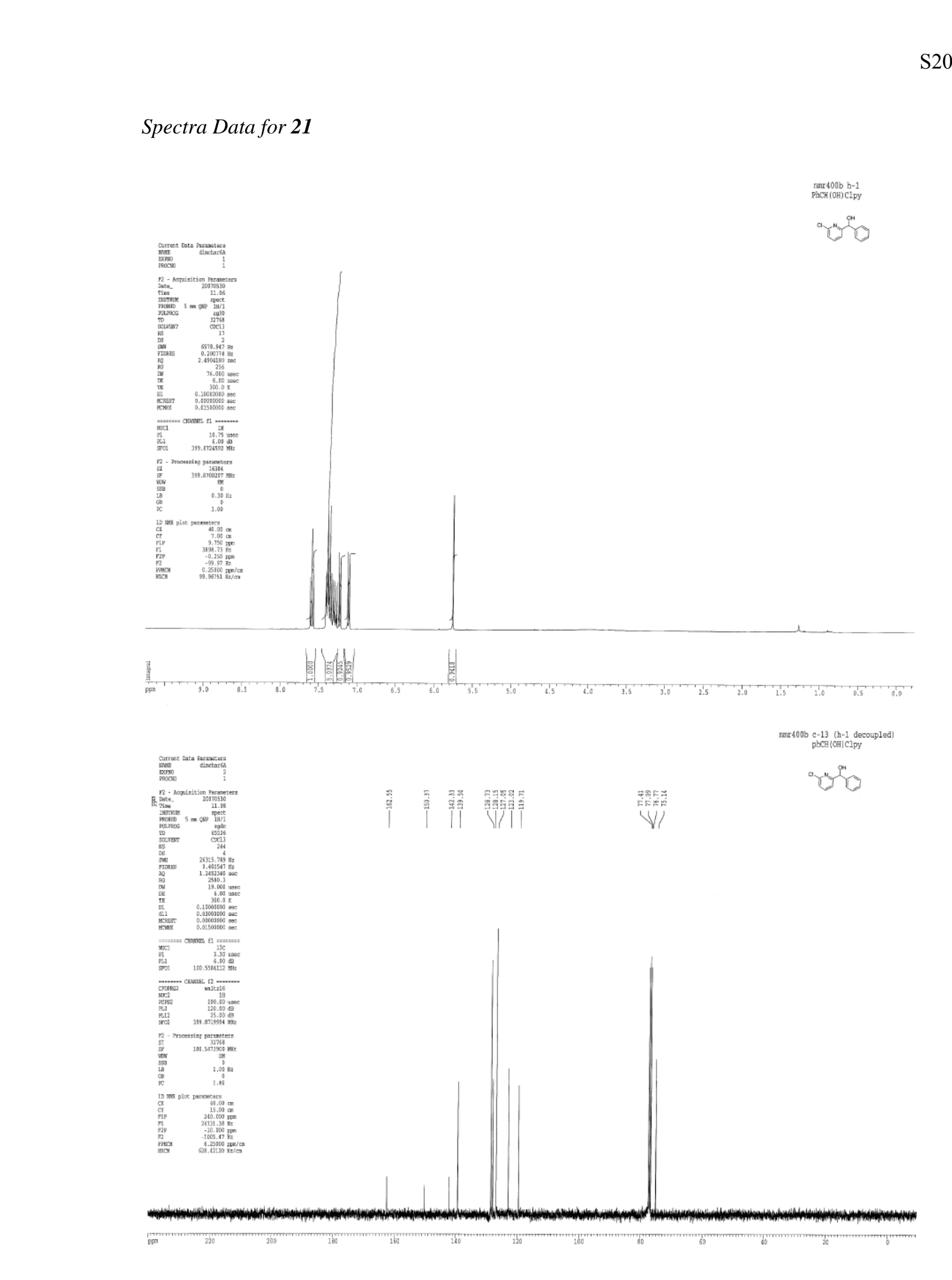

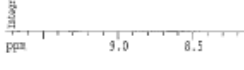

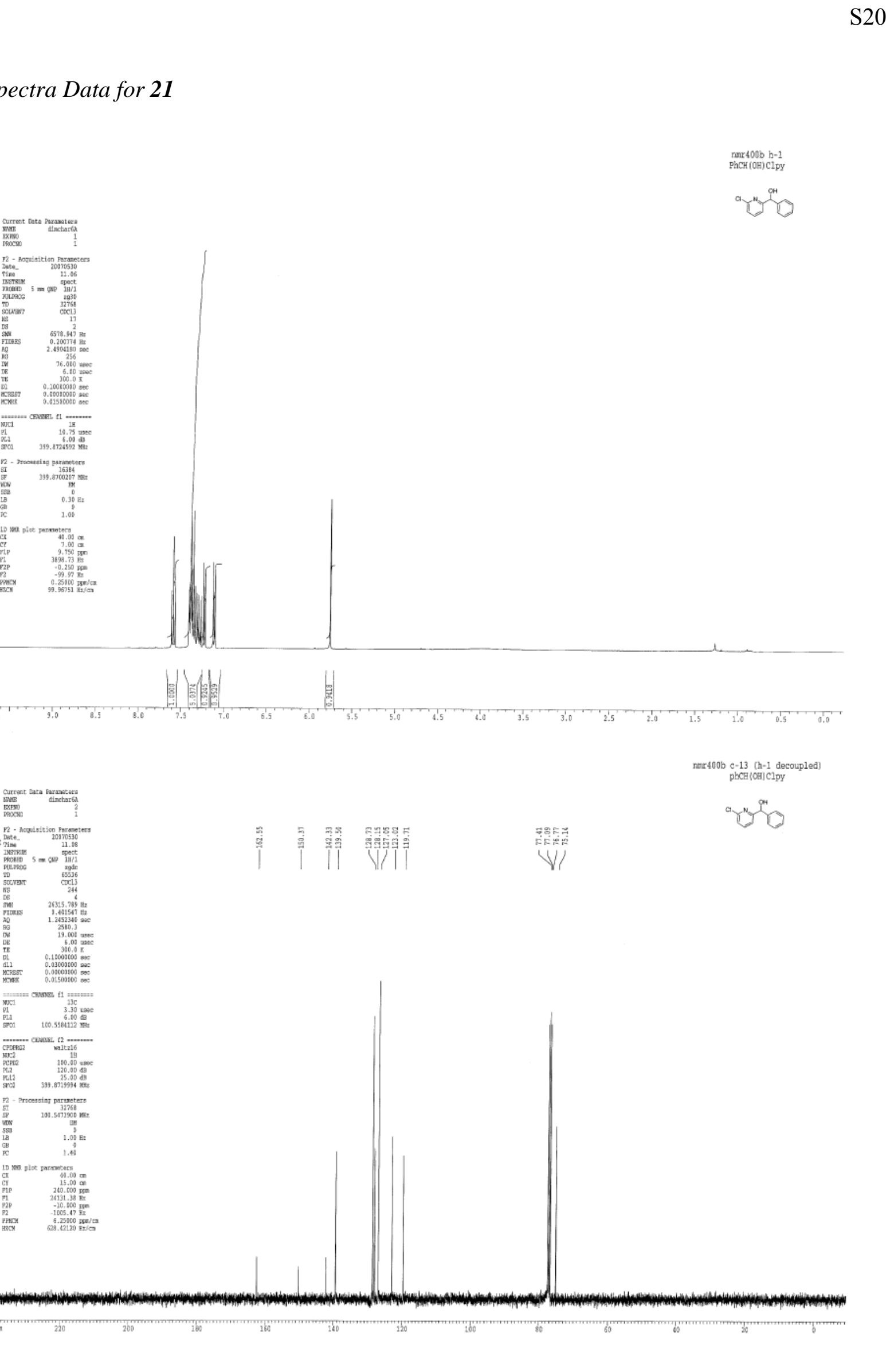

Spectra Data for 21

\section{"10}

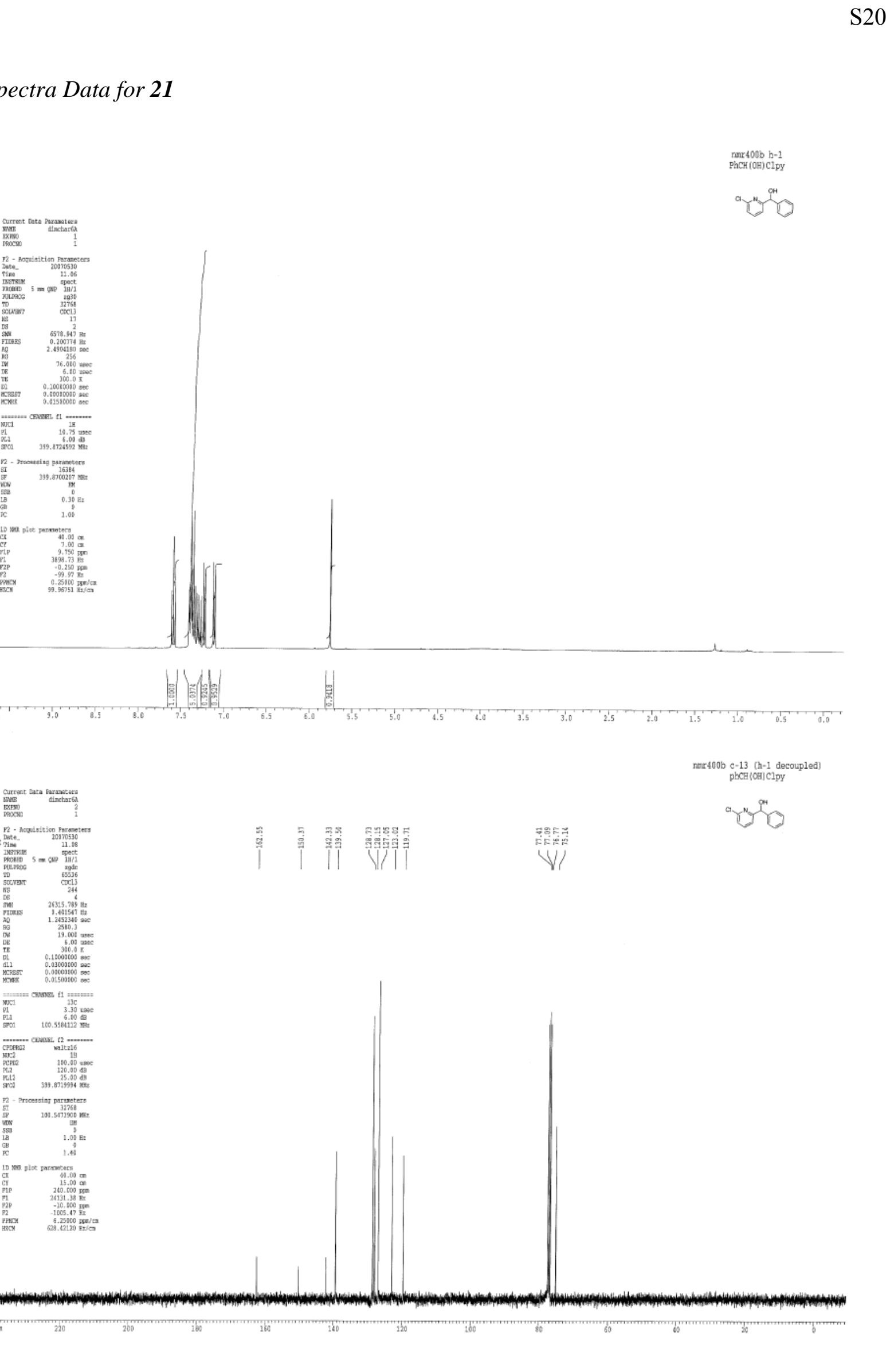


Spectral Data for 5

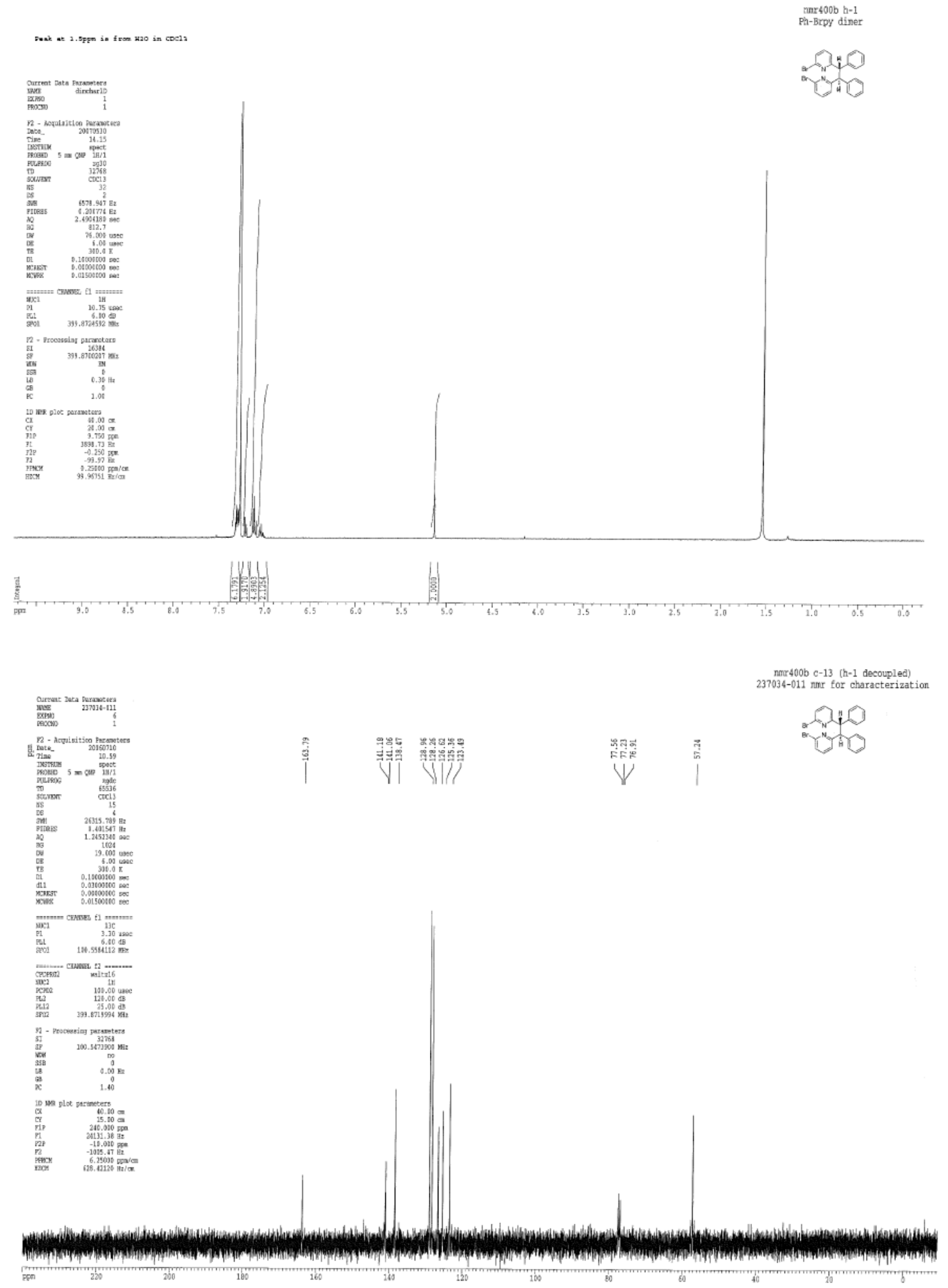




\section{Crystal Structure of 5}

Compound $\mathrm{C}_{24} \mathrm{H}_{18} \mathrm{Br}_{2} \mathrm{~N}_{2}, M_{r}=494.220$, orthorhombic, $P 2{ }_{1} 2{ }_{1} 2_{1}, a=9.0694(5), b=11.7804(6), c=19.4812(11) \AA$, $V=2081.4(2) \AA^{3}, Z=4, D_{x}=1.577 \mathrm{gcm}^{-3}$, monochromatized radiation $\lambda(\mathrm{Mo})=0.71073 \AA, \mu=3.91$ $\mathrm{mm}^{-1}, F(000)=984, T=100^{\circ} \mathrm{K}$. Data were collected on a Bruker CCD diffractometer to a $\theta$ limit of $27.17^{\circ}$ which

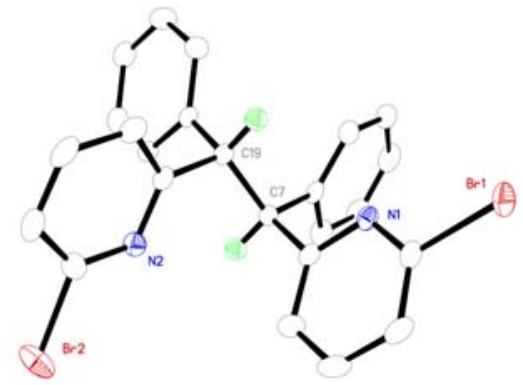
yielded 23314 reflections. There are 4584 unique reflections with 4408 observed at the $2 \sigma$ level. The structure was solved by direct methods (SHELXS-97, Sheldrick, G.M. Acta Crystallogr., 1990, A46, 467-473) and refined using full-matrix least-squares on $F^{2}$ (SHELXL-97, Sheldrick, G.M. SHELXL-97. Program for the Refinement of Crystal Structures. Univ. of Göttingen, Germany). The final model was refined using 253 parameters and all 4584 data. All non-hydrogen atoms were refined with anisotropic thermal displacements. The final agreement statistics are: $R=0.020$ (based on 4408 reflections with $I$ $>2 \sigma(I)), w R=0.051, S=1.10$ with $(\Delta / \sigma)_{\max }<0.01$. The maximum peak height in a final difference Fourier map is $0.544 \mathrm{e} \AA^{-3}$ and this peak is without chemical significance. CCDC 667024 contains the supplementary crystallographic data for this paper. These data can be obtained free of charge from The Cambridge Crystallographic Data Centre via www.ccdc.cam.ac.uk/data_request/cif. 
Spectral Data for 22

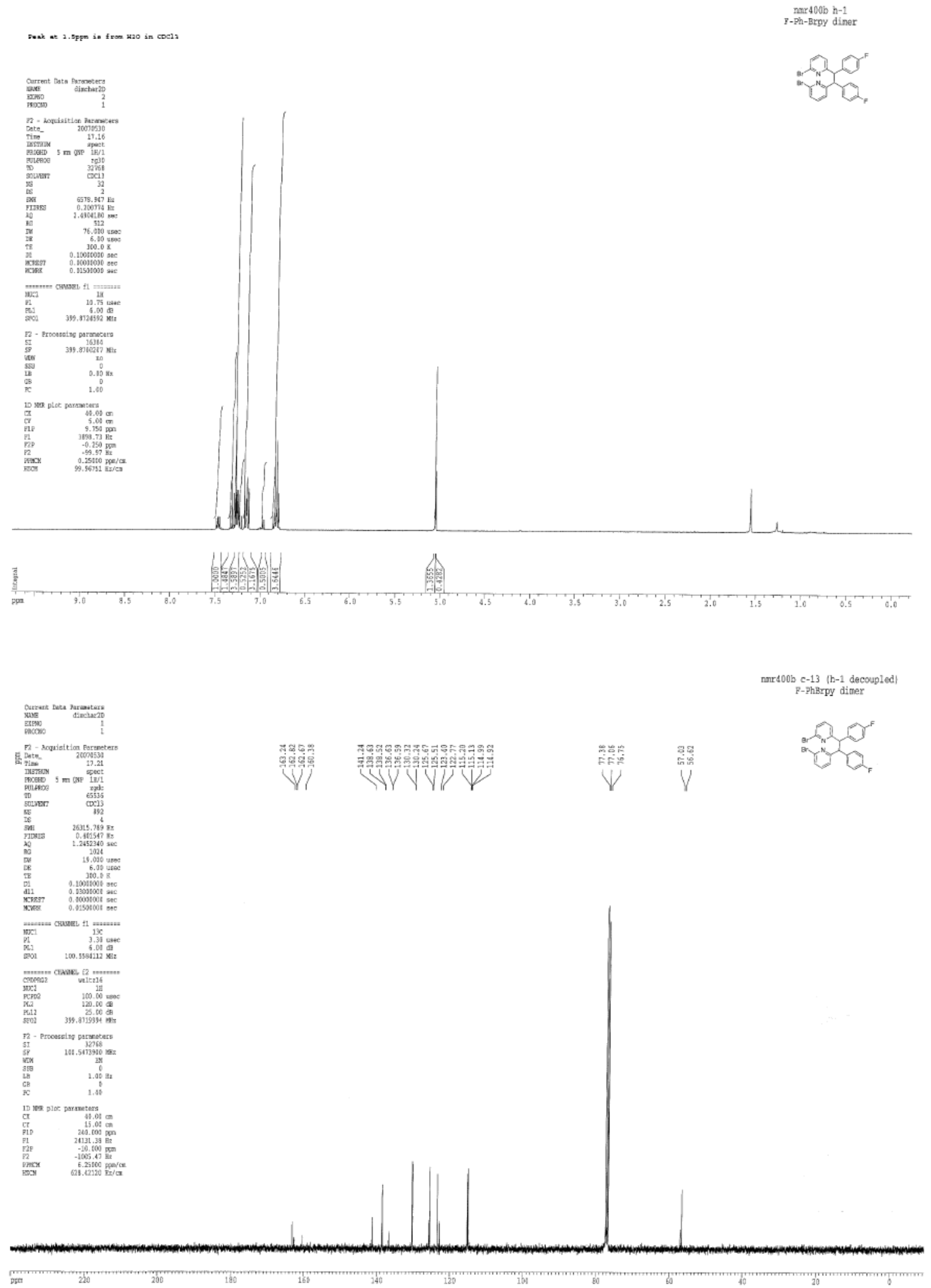


Spectral Data for 23

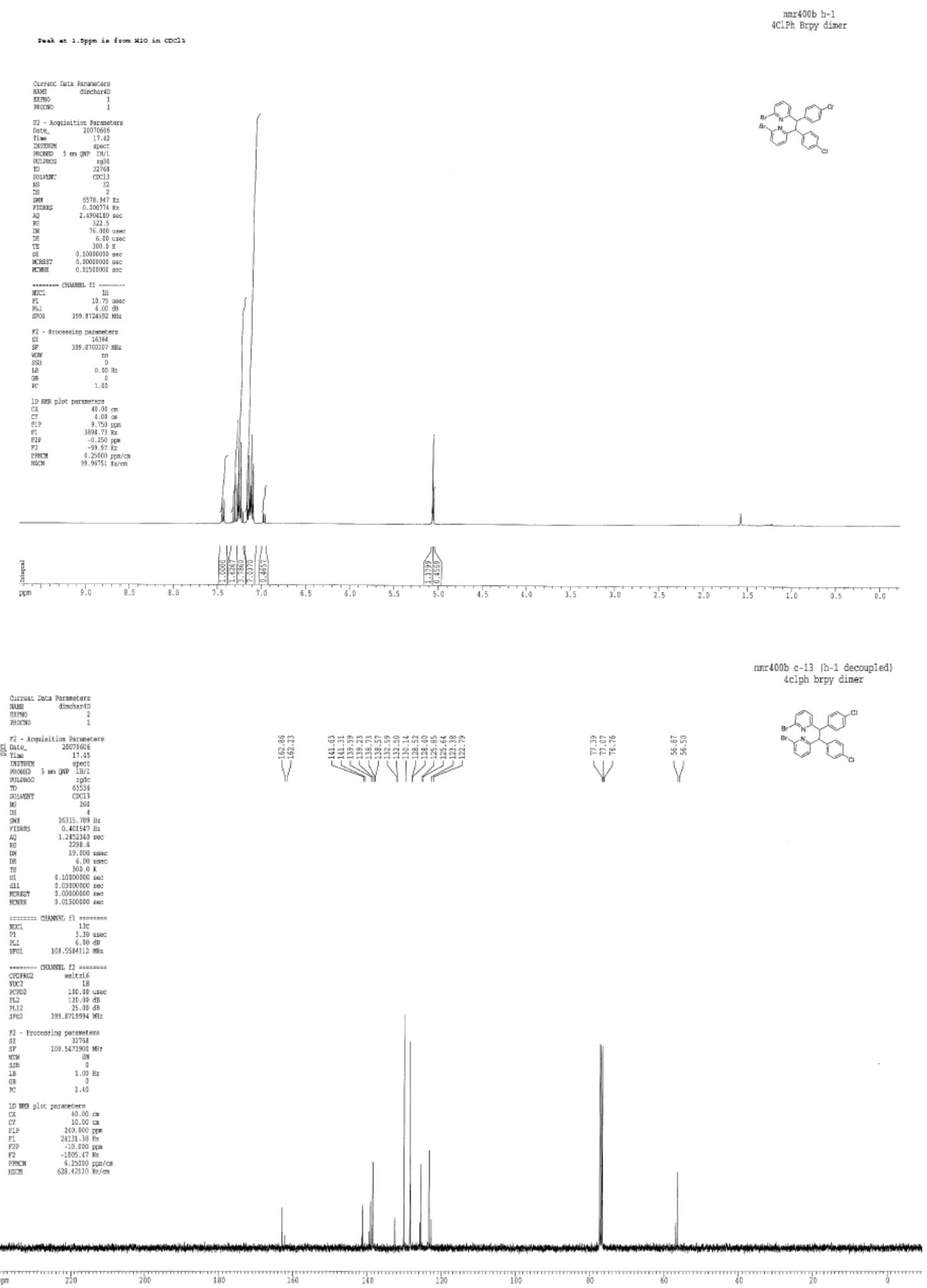


Spectral Data for 24

Peak at $1.5 \mathrm{ppm} 1 \mathrm{~s}$ from $\mathrm{H} 2 \mathrm{O}$ in $\mathrm{CDCl}_{3}$

3-Xe srpy dizer

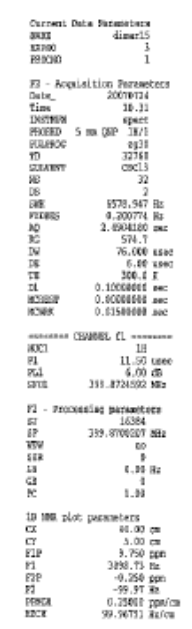

* on

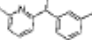
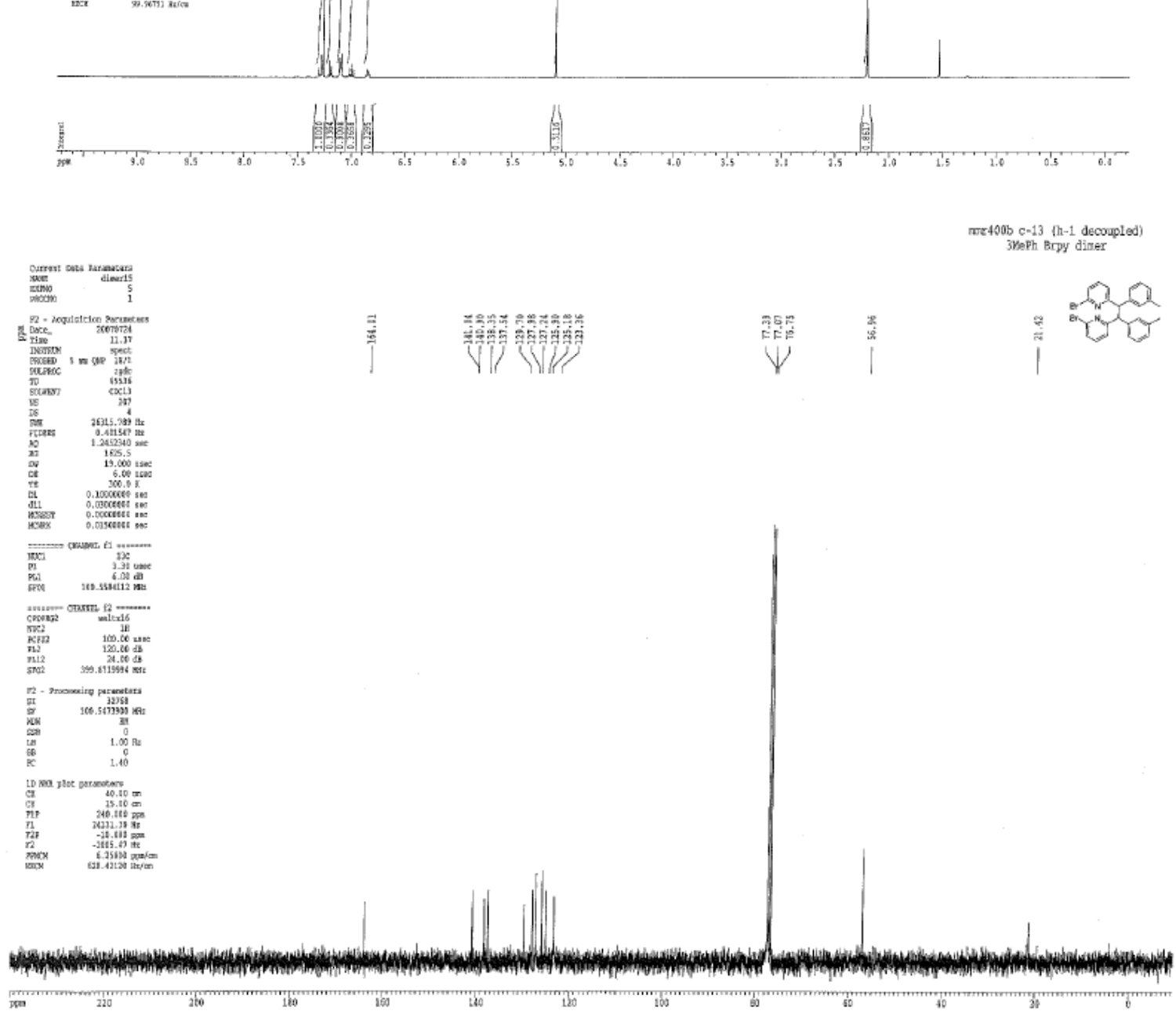
Spectra Data for 25
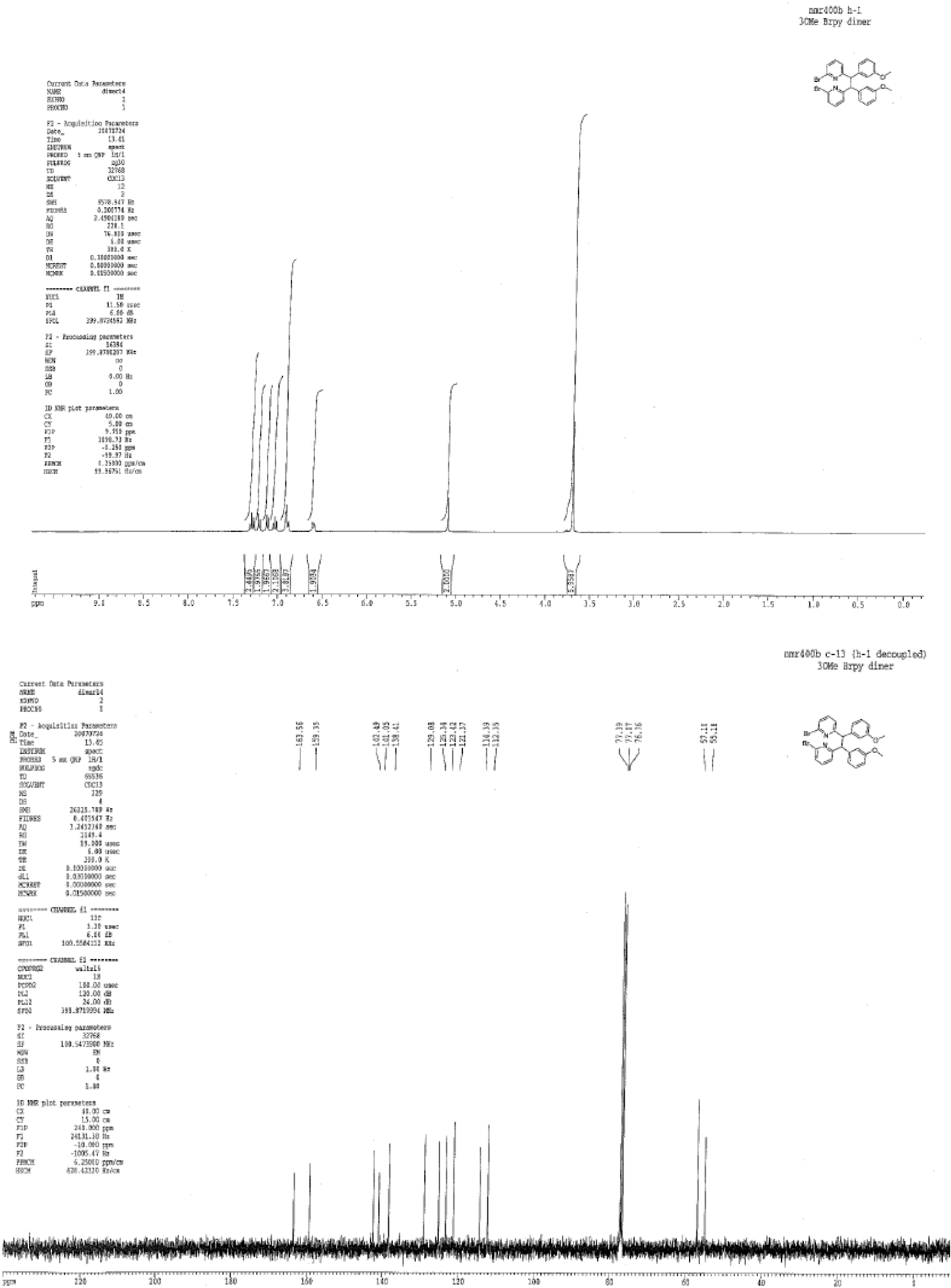
Spectral Data for 29
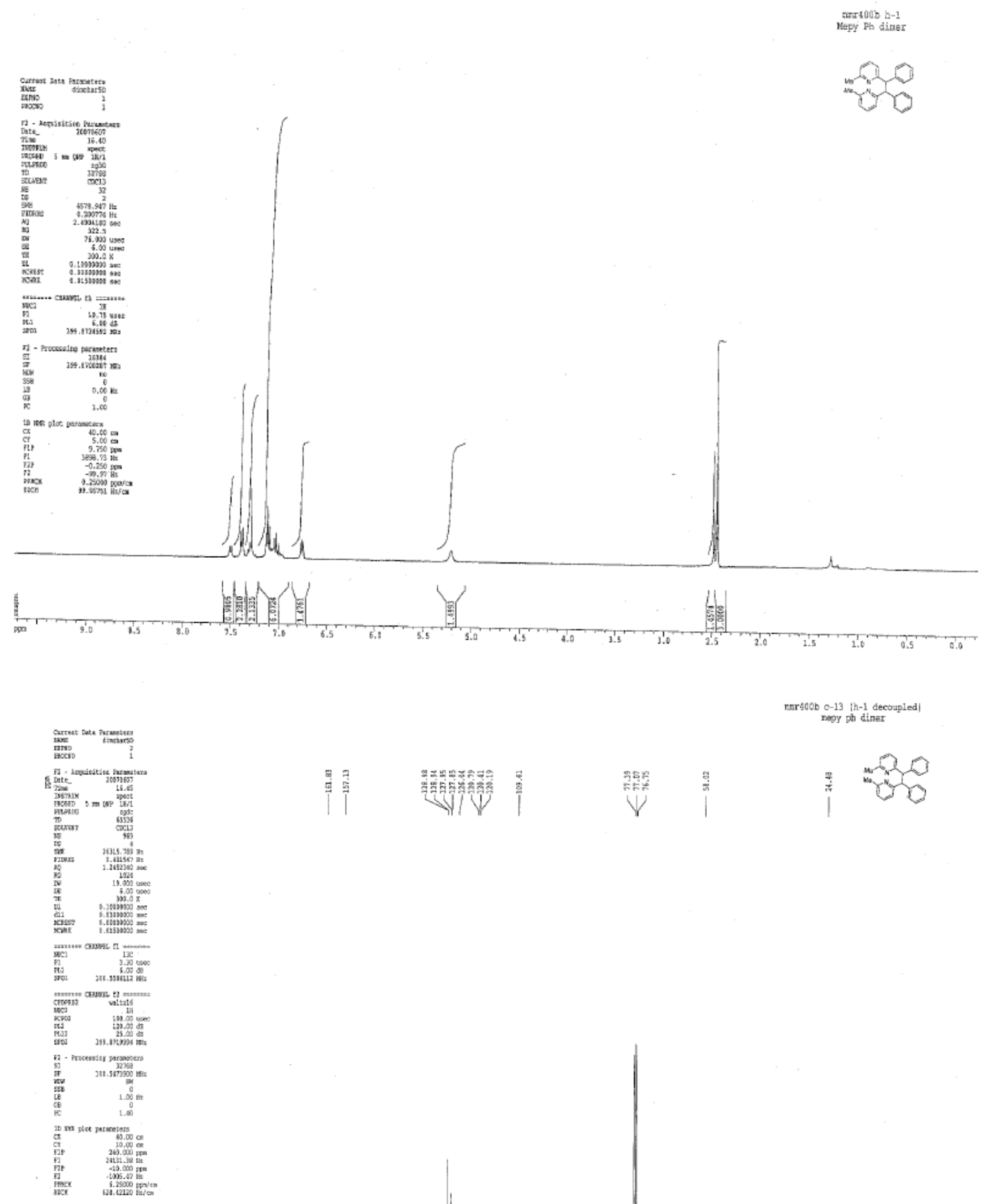

nexpocob o-13 th-1 Gecoupled
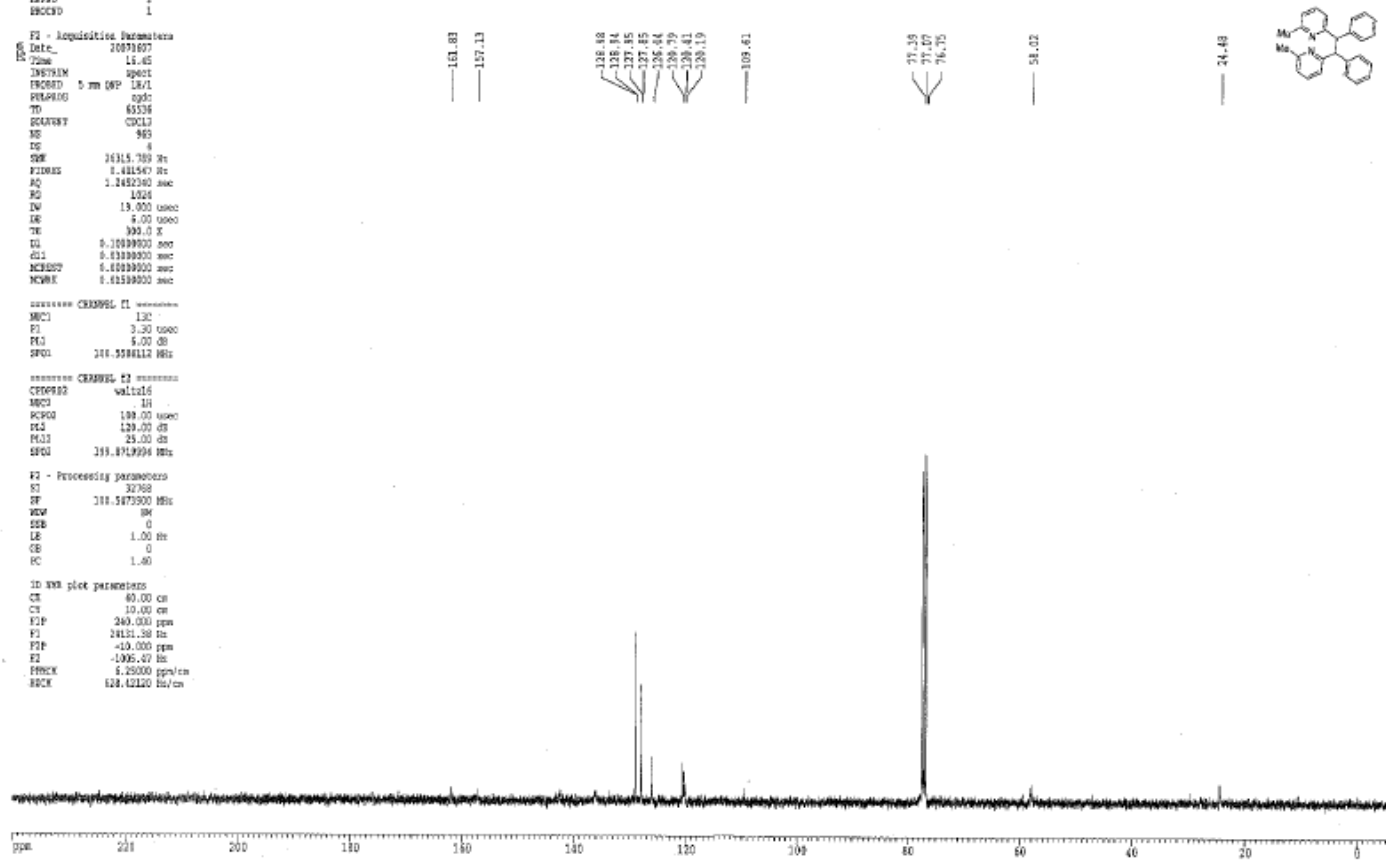

140 
Spectra Data for 30

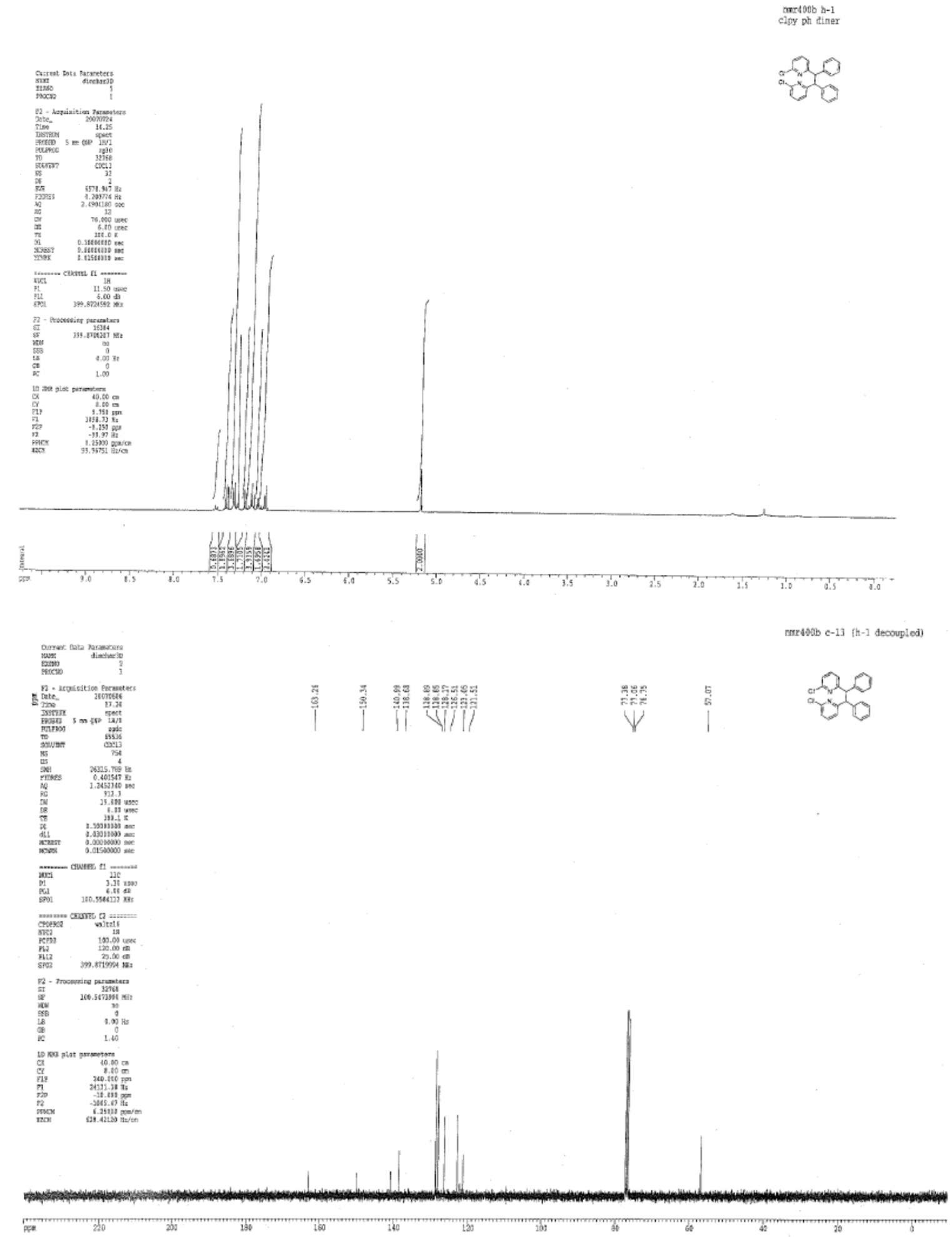


Spectra Data for 32

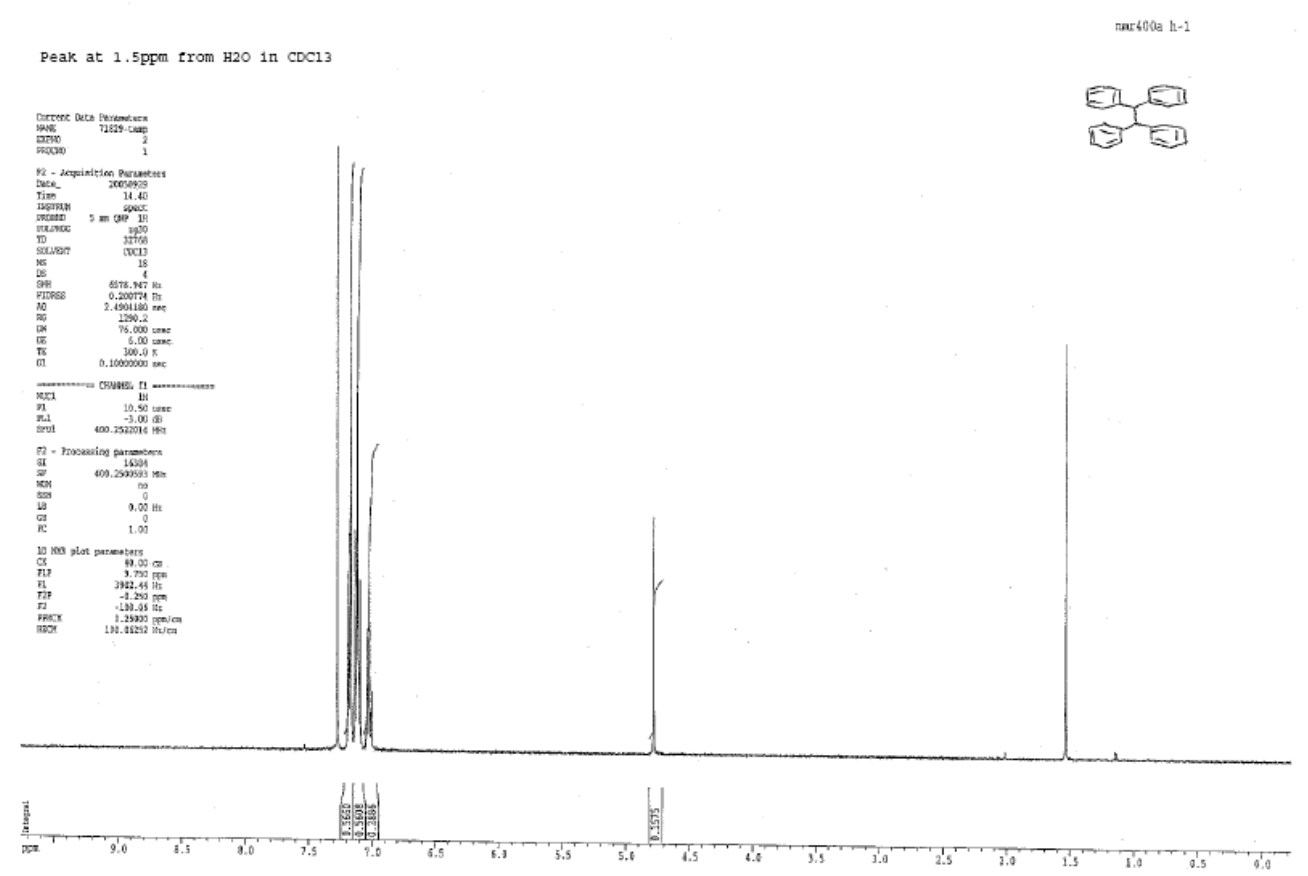

Spectral Data for 33

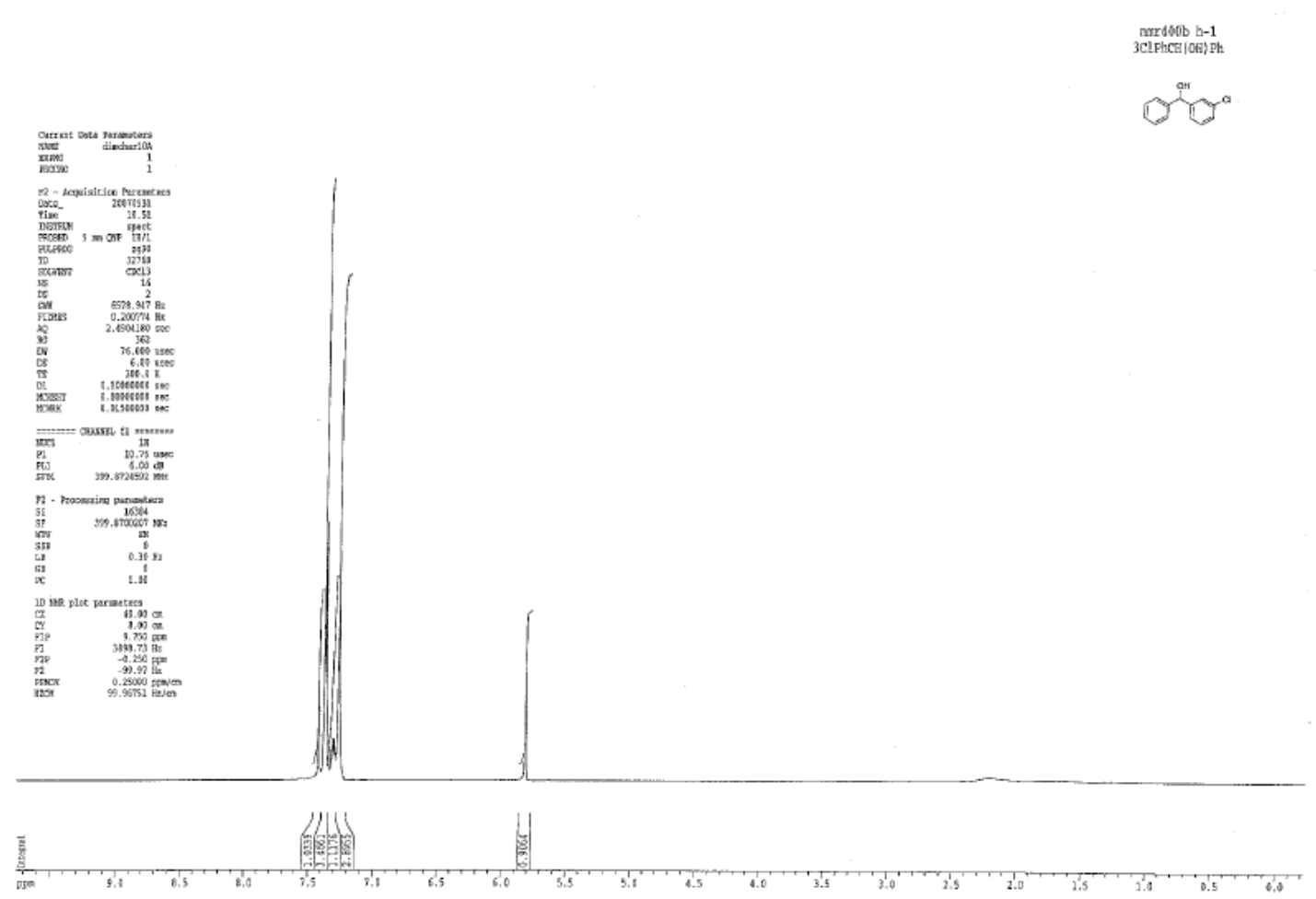


Spectra Data for 34
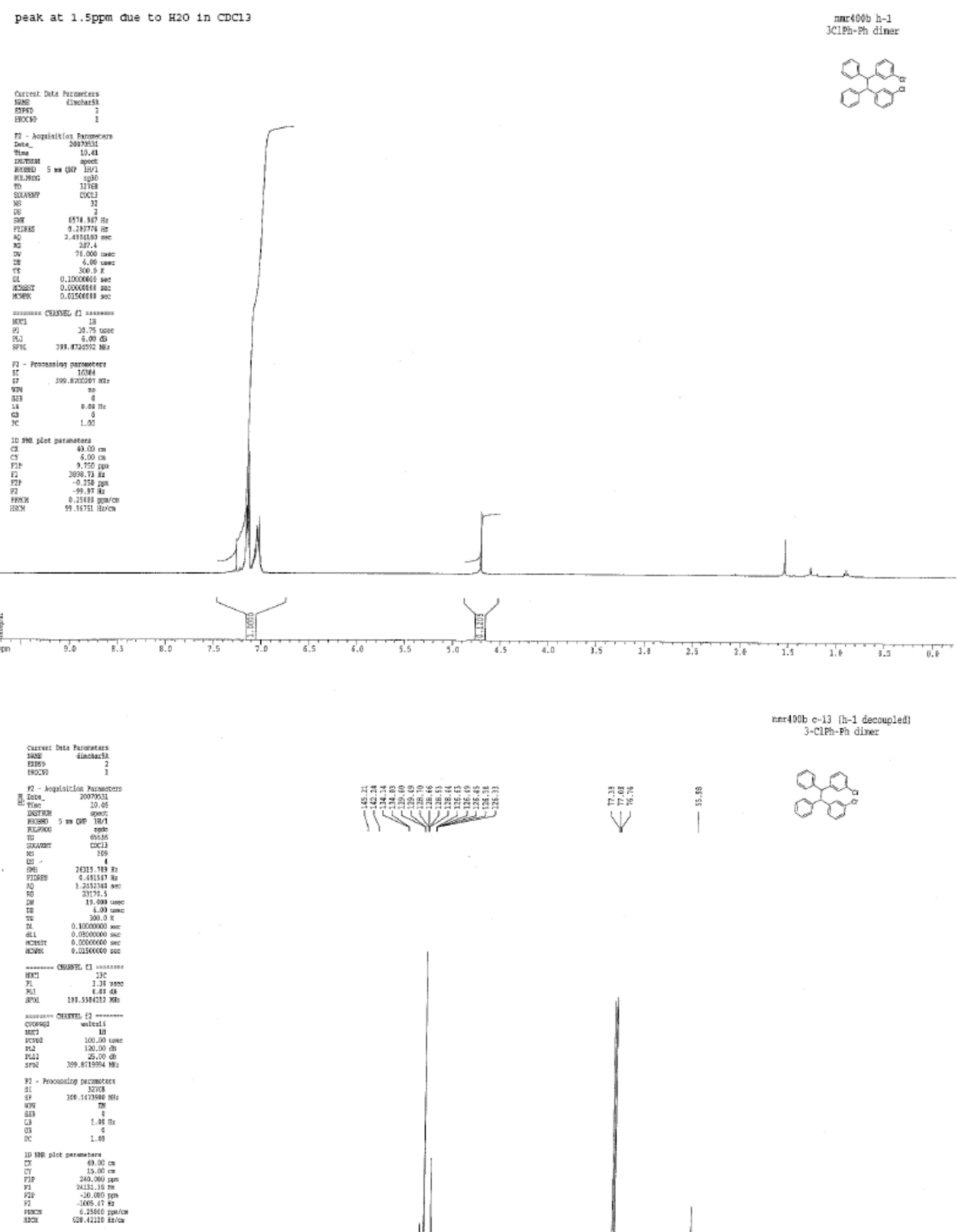